\title{
Large-Eddy Simulations of Surface Heterogeneity Effects on the Convective Boundary Layer During the LITFASS-2003 Experiment
}

\author{
Björn Maronga · Siegfried Raasch
}

Received: 15 July 2011 / Accepted: 14 June 2012 / Published online: 10 July 2012

(C) The Author(s) 2012. This article is published with open access at Springerlink.com

\begin{abstract}
We investigate the impact of observed surface heterogeneities during the LITFASS-2003 experiment on the convective boundary layer (CBL). Large-eddy simulations (LES), driven by observed near-surface sensible and latent heat fluxes, were performed for the diurnal cycle and compare well with observations. As in former studies of idealized one- and two-dimensional heterogeneities, secondary circulations developed that are superimposed on the turbulent field and that partly take over the vertical transport of heat and moisture. The secondary circulation patterns vary between local and roll-like structures, depending on the background wind conditions. For higher background wind speeds, the flow feels an effective surface heat-flux pattern that derives from the original pattern by streamwise averaging. This effective pattern generates a roll-like secondary circulation with roll axes along the mean boundary-layer wind direction. Mainly the upstream surface conditions control the secondary circulation pattern, where the fetch increases with increasing background wind speed. Unlike the entrainment flux that appears to be slightly decreased compared to the homogeneously-heated CBL, the vertical flux of sensible heat appears not to be modified in the mixed layer, while the vertical flux of latent heat shows different responses to secondary circulations. The study illustrates that sufficient time averaging and ensemble averaging is required to separate the heterogeneity-induced signals from the raw LES turbulence data. This might be an important reason why experiments over heterogeneous terrain in the past did not give any clear evidence of heterogeneity-induced effects.
\end{abstract}

Keywords Convective boundary layer · Large-eddy simulation · LITFASS-2003 . Secondary circulation $\cdot$ Surface heterogeneity

B. Maronga $(\varangle) \cdot S$. Raasch

Institut für Meteorologie und Klimatologie, Leibniz Universität Hannover,

Herrenhäuser Str. 2, 30419 Hannover, Germany

e-mail: maronga@muk.uni-hannover.de 


\section{Introduction}

Land-surface heterogeneities can be divided into topographical and land-use heterogeneities that are widely spread over the Earth's surface. Differences in land use represent heterogeneities with a high variability in vegetation, soil texture and wetness. They lead to spatial differences in surface properties such as temperature, humidity and roughness and thus to different surface fluxes of sensible and latent heat as well as momentum. The size of the heterogeneities range from continental scale down to the mesoscale and microscale. In operational weather forecast models, the orography of the land surface is already considered, being a crucial factor for the quality of the forecast. Despite the known effects of large-scale heterogeneities such as land and sea (e.g. the land-sea breeze), it is still uncertain whether heterogeneous land use on the mesoscale and microscale produces significant effects on the atmospheric boundary layer and on the local weather.

In several experimental studies, e.g. LITFASS-98 (Lindenberg Inhomogeneous Terrain Fluxes between Atmosphere and Surface: a long-term Study, Beyrich et al. 2002a,b), LITFASS-2003 (Beyrich and Mengelkamp 2006), the International $\mathrm{H}_{2} \mathrm{O}$ Project (Weckwerth et al. 2004) and SMACEX (Soil Moisture-Atmosphere Coupling Experiment, Kustas et al. 2005), the convective boundary-layer (CBL) characteristics over a heterogeneous land surface have been investigated. However, a heterogeneous effect could hardly be captured.

Over the past two decades, turbulence-resolving large-eddy simulations (LES) have increasingly been used for the investigation of the interaction between surface heterogeneities and the CBL by resolving the bulk of the energy-containing eddies (e.g. Hechtel et al. 1990; Hadfield et al. 1991, 1992; Shen and Leclerc 1995; Avissar and Schmidt 1998; Gopalakrishnan and Avissar 2000; Raasch and Harbusch 2001, hereafter RH01; Letzel and Raasch 2003; Patton et al. 2005, hereafter PSM05; Courault et al. 2007; Huang and Margulis 2009).

The earlier studies investigated small-scale heterogeneities in the order of a few hundreds of metres with no background flow (Hechtel et al. 1990; Hadfield et al. 1991) or with background flow (Hadfield et al. 1992), but found no significant effect on the boundary-layer structure. The later studies of Shen and Leclerc (1995) as well as RH01 used checkerboardlike two-dimensional heterogeneities and reported that the surface heterogeneities in fact must be at least of the size of the boundary-layer height $z_{i}$ to influence the boundary-layer characteristics. The simulations of sinusoidal stripe-like one-dimensional heterogeneity by Avissar and Schmidt (1998) showed the development of secondary circulations, but that a background wind speed of $5 \mathrm{~m} \mathrm{~s}^{-1}$ suffices to eliminate the effect of the surface heterogeneity. RH01 found the secondary circulations to persist even for background wind speeds up to $7.5 \mathrm{~m} \mathrm{~s}^{-1}$ depending on the mean flow orientation relative to their checkerboard inhomogeneity. Letzel and Raasch (2003) found temporal oscillations in the turbulent kinetic energy (TKE) for larger heterogeneity scales that led to an oscillation of the secondary circulation magnitude. Kang (2009) also investigated these temporal oscillations and suggested that the onset of the oscillations might be the start of a break-up process of fluctuations at the scale of the mesoscale heterogeneity to small-scale fluctuations. Gopalakrishnan and Avissar (2000) found that surface heterogeneities with a characteristic length scale of $5 \mathrm{~km}$ decrease the vertical mixing of particles, but increase the horizontal mixing.

PSM05 used one-dimensional soil-moisture heterogeneities in their LES coupled to a soilvegetation-atmosphere transfer (SVAT) model, which incorporates the feedback between secondary circulations and surface fluxes, while most other studies used prescribed surface fluxes. They reported a dependency of the secondary circulations on the scale of the heterogeneity (wavelength $\lambda$ ), $z_{i}$ and the initial state of moisture. Particularly, they found that 
the strongest CBL response to the heterogeneity occurs for $\lambda / z_{i}$ between 4 and 9 , owing to the interaction of the secondary circulations and the induced surface fluxes in the SVAT model. Furthermore, PSM05 found the secondary circulations to contribute up to $70 \%$ (height-dependent) to vertical fluxes. The coupled LES-SVAT study of Courault et al. (2007) investigated the feedback of surface heat fluxes to secondary circulations, and suggested that small-scale heterogeneities (here $\lambda=5 \mathrm{~km}$ ) are able to induce secondary circulations that lead to the horizontal transport of moisture from wet to dry areas. Courault et al. (2007) reported a decrease in the surface sensible heat flux over the dry patch, leading to a modification of the area-averaged surface fluxes. Liu et al. (2011) used checkerboard surface heat-flux patterns in their LES and showed that their heterogeneity-induced circulations break-up after a certain time, when $\lambda / z_{i}$ is small enough due to the increasing boundary-layer depth. This can be traced back to the results of Shen and Leclerc (1995) and RH01. Liu et al. (2011) also reported no significant impact on the profiles of temperature and the sensible heat flux. Kang and Davis (2008) used a spatial filtering method and showed that the vertical mesoscale transport in the presence of large-scale heterogeneities $(\lambda=16 \mathrm{~km}$ and $\lambda=32 \mathrm{~km})$ is negligible compared to the turbulent transport.

Until now these dependencies of secondary circulations on the background flow as well as on the heterogeneity scale have been investigated by means of idealized one- or twodimensional periodic heterogeneities only. The question whether the findings mentioned above are also valid over complex terrain, where heterogeneities of a whole range of scales are superimposed upon each other, or whether secondary circulations develop at all, has not been studied to date. Furthermore the question arises, how secondary circulations vary when simulating a full diurnal cycle, in which the surface forcing as well as $z_{i}$ changes in time.

Some studies reported modifications of the CBL depth in such a way that the mixing layer is deeper over warmer surface patches and thinner over colder patches (e.g. van Heerwaarden and de Arellano 2008, hereafter HA08; Fesquet et al. 2009). The reason for the spatial variation of $z_{i}$ in the study of HA08 was associated with rising secondary circulation updrafts that increase the entrainment above the warm patches. Furthermore they reported changes in the entrainment rate, but attributed this finding to differences in their model spin-up or as an effect of horizontal averaging (Lilly 2002). Moreover, HA08 found the specific humidity variance in the entrainment zone to be larger than under homogeneous conditions and thus suggested that cloud formation might be enhanced over heterogeneous surfaces.

Contrary to most previous studies, Huang and Margulis (2009) recently used observed surface fluxes from SMACEX in their LES to investigate the CBL development over irregular surface heterogeneities. They found increasing entrainment rates for increasing surface heterogeneity scales. Nevertheless their results displayed atypical large entrainment fluxes, whose origin was not discussed. The question of whether area-averaged vertical fluxes are modified due to the effects of observed heterogeneous surfaces has thus not been sufficiently clarified to date.

The present study takes a step forward by explicitly resolving the secondary circulations over a complex non-periodic irregular heterogeneity. Effects of the repetition of the heterogeneity due to cyclic model boundaries are considered. A series of LES was carried out with surface boundary conditions derived from measurements from the LITFASS-2003 experiment (Beyrich and Mengelkamp 2006). The experimental site $\left(400 \mathrm{~km}^{2}\right)$ was located to the south-east of Berlin (Germany) and exhibited moderately heterogeneous land use as is typically found in Central Europe, including lakes, patches of forest and several agricultural fields. This study attempts to identify secondary circulations and their impact on the CBL over this particular surface heterogeneity. It is found that secondary circulations are much more complex compared to previous studies of regular heterogeneities and that it is difficult 
to relate the secondary circulation pattern to the surface heat-flux pattern. The correlation between these patterns is analyzed. Additionally, the impact on the entrainment at the top of the CBL and the effect on area-averaged fluxes are studied. Some preliminary results that have been presented by Uhlenbrock et al. (2004) inspired us in large part to develop the present study. Preliminary results have also been shown in Beyrich and Mengelkamp (2006) and Foken et al. (2009), but are partly revised herein.

The paper is organized as follows: Sect. 2 describes the LES model, the implementation of irregular surface heterogeneities and the driving mechanism for the simulations as well as the determination of secondary circulations over such complex heterogeneities. Simulation results are presented in Sect. 3, while Sect. 4 gives a summary and a brief outlook on further studies.

\section{LES Model and Simulations Set-Up}

\subsection{The Parallelized LES Model PALM}

The PArallelized LES Model (PALM) was used for the present study (Raasch and Etling 1998; Raasch and Schröter 2001). All simulations were carried out using cyclic lateral boundaries, and Monin-Obukhov similarity theory (MOST) was applied between the surface and the first computational grid level. At the beginning of each simulation, a random generator is used to create small perturbations of the velocity field to initiate turbulence.

PALM has been widely applied to study different flow regimes in the homogeneous (Schröter et al. 2000) and heterogeneous heated CBL (e.g. RH01; Letzel and Raasch 2003; Steinfeld et al. 2008), in the weakly stable boundary layer (Beare et al. 2007; Steinfeld et al. 2007) as well as under neutral conditions (Letzel et al. 2008).

\subsection{Determining Secondary Circulations Above Irregular Surface Heterogeneities}

In simulations of the flow over real world irregular heterogeneities, cyclic boundary conditions affect the secondary circulations, because the heterogeneity-induced pattern is peridiodically repeating, which does not occur in reality. Hence, in the vicinity of horizontal boundaries, the flow is exposed to an erroneous surface forcing, especially if the mean flow is directed from the boundaries to the inner model domain. One method to overcome this problem is to restrict data analysis to an inner area of the model domain that is located as far away as possible from the horizontal boundaries. This normally requires enlarging the model domain in order to provide so-called buffer zones around the analysis area. Within the buffer zones, observed surface heterogeneities have to be provided as surface boundary conditions. For increasing background wind speed, increasing buffer zones must be defined in order to account for advection from greater distances.

Prabha et al. (2007) investigated secondary circulations over an irregular heterogeneity and attached 1-km wide buffer zones with a homogeneous surface, which we find to be inadequate. Huang and Margulis (2009) simulated irregular surface heterogeneities that were periodically horizontally repeating; this was valid for their purpose, but led to unrealistic secondary circulation patterns. The results of our sensitivity study demonstrate that the choice of a sufficient buffer zone can avoid such systematical shortcomings. The required size of the buffer zone increases for increasing prescribed background wind speed and can easily reach $20 \mathrm{~km}$ and more. For a detailed description of the sensitivity study, see Appendix B. 
For separating the secondary circulations from the primary circulation, i.e. the random turbulent convection, idealized studies have commonly used combined temporal and spatial averaging (e.g. RH01; PSM05). In the case of one-dimensional heterogeneities (stripes) or two-dimensional heterogeneities (checkerboard pattern), the flow was averaged over repeating surface patches (phase average). For complex observed heterogeneities another approach is necessary. A phase average cannot be applied because patterns have no symmetry axis and do not repeat. In order to simulate the diurnal cycle, as in the present study, time averaging is also limited, because the CBL does not reach a steady state due to the changes in the surface fluxes as well as the increase of $z_{i}$ in the course of a day. Instead, we thus used a combination of time-averaging and ensemble-averaging methods (1-h time average and eight ensemble runs). The ensemble-averaging method is described in detail in Appendix A.

By means of this ensemble-averaging method, the heterogeneity-induced part $\Phi_{\mathrm{hi}}$ of a quantity $\Phi$ for a fixed 1-h averaged time interval, e.g. 1200-1300 UTC, 1300-1400 UTC, etc., can be calculated as follows:

$$
\bar{\Phi}_{\mathrm{hi}}(x, y, z, t)=\tilde{\bar{\Phi}}(x, y, z, t)-\langle\bar{\Phi}\rangle(z, t),
$$

where $\widetilde{\Phi}$ and $\bar{\Phi}$ represent the ensemble and the time averages, respectively, and $\langle\Phi\rangle$ denotes the horizontal mean of $\Phi$. For the fixed averaging interval it holds that $\bar{\Phi}_{\mathrm{hi}} \approx \Phi_{\mathrm{hi}}$ and hence we hereafter simply omit the overbar. The separation of atmospheric variables after PSM05 can be rewritten as:

$$
\Phi(x, y, z, t)=\langle\Phi\rangle(z, t)+\Phi_{\mathrm{hi}}(x, y, z, t)+\Phi_{\mathrm{S}}(x, y, z, t) .
$$

The small-scale part $\Phi_{\mathrm{S}}$ is also called background turbulence (PSM05) or turbulent part, which represents the primary circulation of randomly distributed convective plumes.

The described separation of scales in Eq. 2 can also be derived for domain- and timeaveraged vertical fluxes:

$$
\langle\overline{w \Phi}\rangle(z)=\overline{\langle w\rangle(z)\langle\Phi\rangle(z)}+\left\langle w_{\mathrm{hi}} \Phi_{\mathrm{hi}}\right\rangle(z)+\left\langle\overline{w_{\mathrm{s}} \Phi_{\mathrm{s}}}\right\rangle(z),
$$

where $\langle\overline{w \Phi}\rangle$ is the horizontally- and temporally-averaged total flux that can be decomposed into a global part $\overline{\langle w\rangle(z)\langle\Phi\rangle(z)}$, a heterogeneity-induced part $\left\langle w_{\mathrm{hi}} \Phi_{\mathrm{hi}}\right\rangle(z)$ (hereafter $\left.\overline{\langle\Phi \Phi}\right\rangle_{\mathrm{hi}}$ ) and a turbulent part $\left\langle\overline{w_{\mathrm{s}} \Phi_{\mathrm{s}}}\right\rangle(z)$ (hereafter $\langle\overline{w \Phi}\rangle_{\mathrm{s}}$ ). Due to cyclic boundaries and the constraint that the equation of continuity for incompressible fluids must be valid, the horizontally-averaged vertical velocity $\langle w\rangle$ in our LES is zero and thus the global part in Eq. 3 vanishes.

In order to determine the effect of surface heterogeneities on $z_{i}$, we calculated a local boundary-layer height from the local potential temperature $(\theta)$ profile following Sullivan et al. (1998):

$$
z_{i}(x, y)=z
$$

where $\frac{\partial \theta(x, y, z)}{\partial z}$ for given $x$ and $y$ is a maximum. Sullivan et al. (1998) showed that the areaaveraged $z_{i}$ is slightly modified compared to non-local methods. For scaling purposes, we follow this local gradient method instead of calculating $\left\langle z_{i}\right\rangle$ from area-averaged profiles.

\subsection{Implementation of the Surface Heterogeneity During LITFASS-2003}

The LITFASS area $\left(A_{\mathrm{L}}\right)$ can be divided into several land-use classes that have different characteristics. The distribution of these characteristics follows the actual land-surface properties. The map in Fig. 1 (see also Beyrich and Mengelkamp 2006, Figs. 2, 3) shows the land-use 


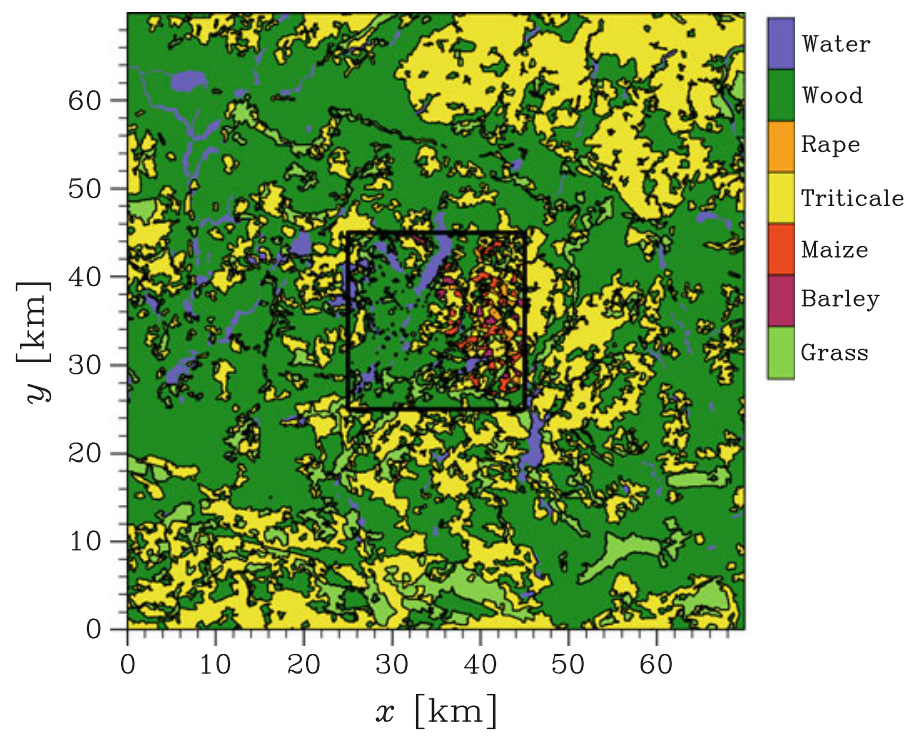

Fig. 1 Distribution of land-use classes in $A_{\mathrm{L}}$ (black box) and surroundings as used in the LES. The classes were derived from the CORINE dataset

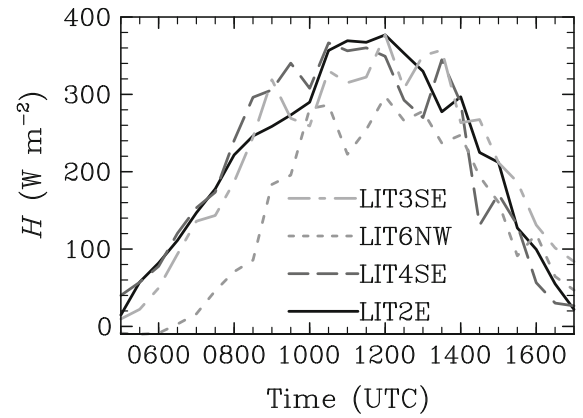

(a) Sensible heat flux

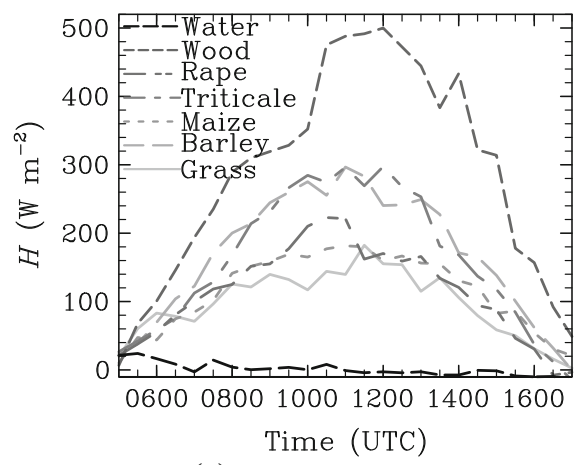

(c) Sensible heat flux

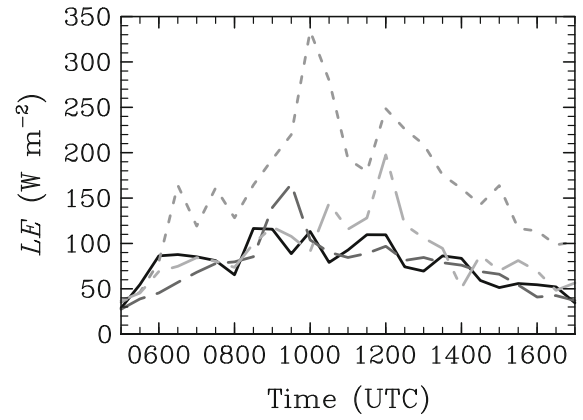

(b) Latent heat flux

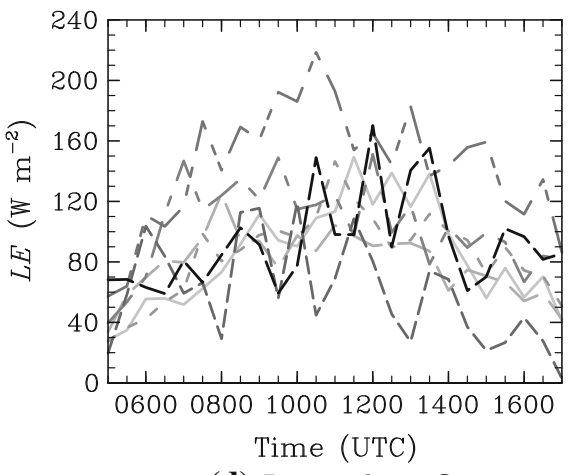

(d) Latent heat flux

Fig. 2 Time series of the prescribed fluxes of sensible and latent heat at the surface: $\mathbf{a}$ and $\mathbf{b}$ are the horizontal mean values as they were used in the homogeneous control runs, while $\mathbf{c}$ and $\mathbf{d}$ are the particular fluxes for different land-use classes for case LIT2E 


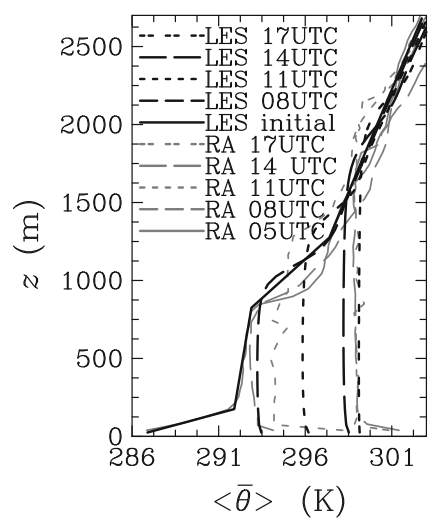

(a) $\operatorname{LIT} 2 \mathrm{E}$

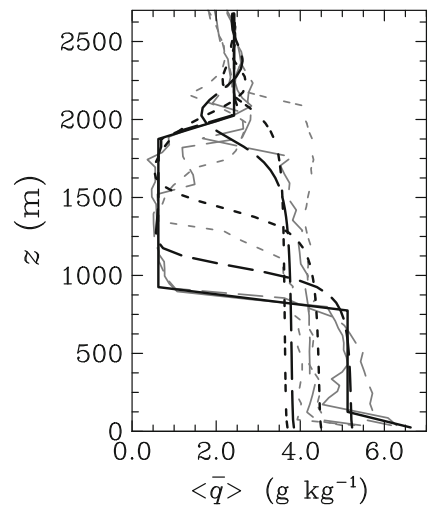

(c) LIT2E

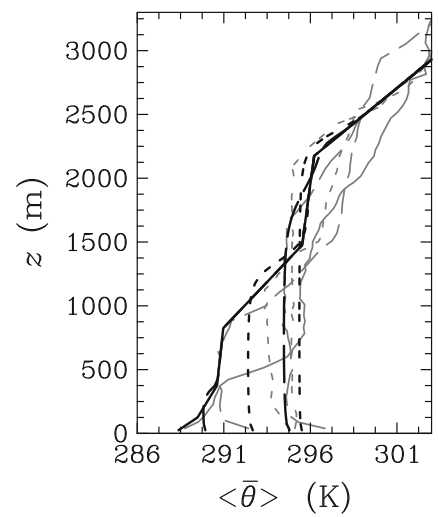

(b) LIT6NW

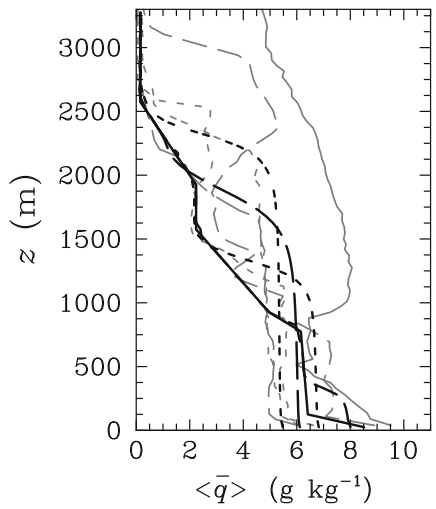

(d) LIT6NW

Fig. 3 Profiles of potential temperature $(\mathbf{a}, \mathbf{b})$ and specific humidity $(\mathbf{c}, \mathbf{d})$ for cases LIT2E (left) and LIT6NW (right) in the course of the day. The LES profiles are domain- and time-averaged (black, 10-min mean), while radiosonde data are shown by grey lines

classes for $A_{\mathrm{L}}$ and the surrounding area, derived from a modified CORINE ${ }^{1}$ dataset. We determined the heterogeneity for an area of size $70 \mathrm{~km} \times 70 \mathrm{~km}$, centred around $A_{\mathrm{L}}$, which is of size $20 \mathrm{~km} \times 20 \mathrm{~km}$. The CORINE dataset contains 44 different land-use classes from which 16 were found to be dominant in the greater LITFASS area. For simplification, the classes "pastures" and "natural grasslands" were merged into the class "grass". The forest patches "mixed forest", "broad-leaved forest" and "coniferous forest" as well as artificial areas (such as settlements) were merged into the class "forest". The latter is reasonable since settlements exhibit similar fluxes of sensible and latent heat as well as roughness properties as forest areas. Agricultural fields were treated as triticale/rye, since this was the dominant type in the area. For $A_{\mathrm{L}}$ itself, the tilled fields were observed during the experiment in 2003, subdivided into land-use classes and mapped on the CORINE dataset. The final map is shown in Fig. 1, containing seven different land-use classes: water, wood, rape, triticale, maize, barley and grass.

1 Abbr. "Coordinated Information on the European Environment", published by the European Environment Agency (http://www.eea.europa.eu/publications/COR0-landcover). 
Table 1 Case definition and results of the LITFASS-2003 simulations

\begin{tabular}{lllllll}
\hline Case & \multicolumn{2}{l}{ Simulation time (UTC) } & $f f\left(\mathrm{~m} \mathrm{~s}^{-1}\right)$ & $d d\left(^{\circ}\right)$ & Domain $(\mathrm{km} \times \mathrm{km})$ & $\left\langle z_{i}\right\rangle \max (\mathrm{m})$ \\
\cline { 2 - 6 } & Start & End & & & \\
\hline LIT2E (May 30) & 0500 & 1700 & 2.0 & 90 & $40 \times 40$ & 2200 \\
LIT4SE (June 2) & 0500 & 1700 & 4.0 & 113 & $48 \times 48$ & 3050 \\
LIT6NW (June 13) & 0430 & 1700 & 6.0 & 320 & $56 \times 56$ & 2400 \\
LIT3SE (June 17) & 0430 & 1700 & 3.2 & 110 & $48 \times 48$ & 2400
\end{tabular}

$f f$ and $d d$ are the prescribed geostrophic wind speed and direction, respectively. The domain size refers to the horizontal extent of the model. $\left\langle z_{i}\right\rangle_{\max }$ is the maximum boundary-layer depth during the simulation

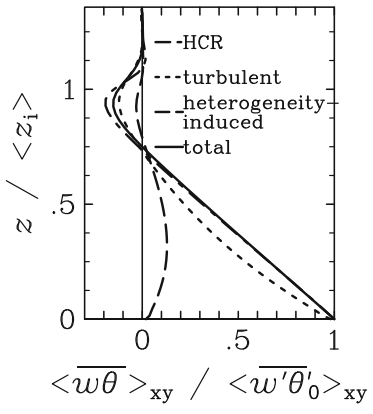

(a) LIT2E

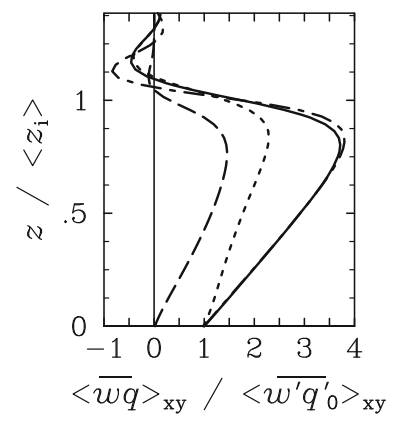

(d) LIT2E

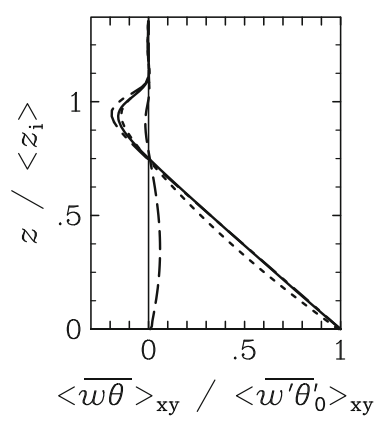

(b) LIT3SE

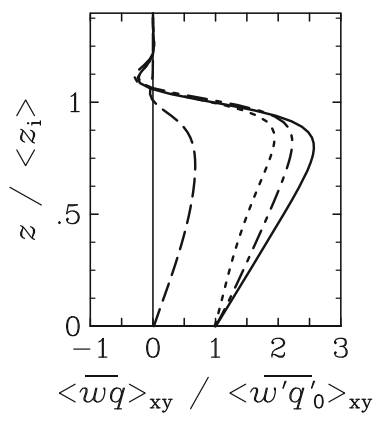

(e) LIT3SE



(c) LIT6NW

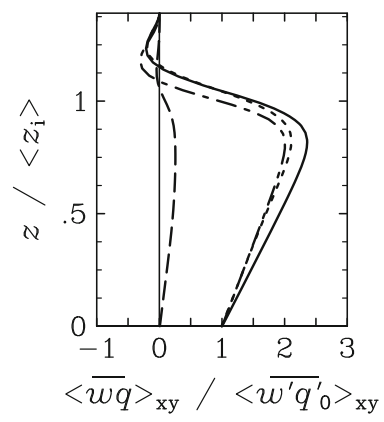

(f) LIT6NW

Fig. 4 Normalized profiles of the vertical kinematic heat fluxes (a-c) and moisture fluxes (d-f) for LIT2E, LIT3SE and LIT6NW at 1300 UTC. The total fluxes (solid black lines) are plotted together with their heterogeneity-induced (dashed lines) and turbulent contributions (short dashed lines). Additionally the total flux from the respective homogeneous control runs are plotted (dot-dashed lines). All fluxes have been time-averaged over $1 \mathrm{~h}$ and normalized by the prescribed surface fluxes

\subsection{Initialization and Forcing of the Simulations}

During the LITFASS-2003 experiment, several energy balance stations as well as other measurement systems, including a 99-m tower (see Beyrich and Mengelkamp 2006, Table 1) were located in the fields of different land use (see Beyrich and Mengelkamp 2006, Fig. 4) The quality assurance showed that the eddy-covariance measurements were representative for the respective land-use class (Beyrich et al. 2006; Mauder et al. 2006). Heinemann and 
Kerschgens (2006) simulated the full experiment period with a mesoscale model and came to the same conclusion. The surface fluxes from the local measurements were thus used as the driving surface fluxes for all patches of the same type of land use. Local differences of plant characteristics were not considered. The flux data were available half-hourly. To avoid discontinuities in the model forcing, the values were linearly interpolated in time. Since usage of a SVAT model would have required the input of several local vegetation and soil parameters, which were not available, prescribing observed fluxes was found to be the best approach for the simulations.

Besides the surface fluxes, the heterogeneity is characterized by a roughness length $z_{0}$, which was estimated by $z_{0} \approx 0.1 \times$ crop height (after Shuttleworth et al. 1997), where the mean crop height for the respective land-use classes was observed during the measurement period. The canopy layer was not explicitly simulated and the flat orography in $A_{\mathrm{L}}$ was neglected.

Initial values of surface temperature and humidity were taken for each land-use class from the measurements at the energy balance stations. Initial profiles of temperature and humidity were derived from radiosonde data and used for the entire model domain, and the resulting profiles were subsequently recomposed by piecewise linear segments. Since the radiosondes did not provide data for height levels below $112 \mathrm{~m}$, a linear interpolation was carried out between the heterogeneous surface temperature (as well as humidity) and the top of the nearsurface temperature inversion in the morning hours. The top of the inversion varied for the simulated days between 75 and $175 \mathrm{~m}$ and was obtained from single point measurements, which were assumed to be representative of the entire area.

A one-dimensional version of the PALM code, with fully-parametrized turbulence using a mixing-length approach after Blackadar (1997) and stationary temperature and humidity profiles, was used for precursor simulations to generate steady-state wind profiles as initialization for the LES. The forcing geostrophic winds in the precursor runs (see Table 1) were chosen such that the steady-state wind profiles were in good agreement with measurements from radiosondes, a tropospheric wind profiler as well as with the $99-\mathrm{m}$ tower measurements.

\subsection{Domain Sizes}

As discussed in Sect. 2.2, it is compulsory to extend the model domain with buffer zones around $A_{\mathrm{L}}$. Therefore the total horizontal model domain $(x \times y)$ varied between $40 \mathrm{~km} \times 40 \mathrm{~km}$ and $56 \mathrm{~km} \times 56 \mathrm{~km}$ (see Table 1). Due to this large domain and the fact that nine simulations (eight ensemble runs, one homogeneous control run, see below) had to be carried out for each case, a grid spacing of $100 \mathrm{~m}$ for the horizontal $(\Delta x, \Delta y)$ and $50 \mathrm{~m}$ for the vertical direction $(\Delta z)$ was chosen in order to reduce the computational demands. About 80 grid points were used in the vertical direction. The vertical grid was stretched well above the inversion in order to further reduce the computational load. Within the scope of the present study, we also simulated three high-resolution cases (homogeneous surface, heterogeneous surface, heterogeneous surface with topography) with $\Delta x, \Delta y=20 \mathrm{~m}$ and $\Delta z=10 \mathrm{~m}$ to prove that our grid resolution is sufficient to resolve the secondary circulations. In the case of the simulations with topography, the elevation was calculated from the real terrain height plus the height of the trees (at those areas with tall vegetation in $A_{\mathrm{L}}$ ). We adjusted the roughness length for these forest areas instead of using a zero-plane displacement. The results of these simulations showed that higher resolution or topography does not have a significant effect on the secondary circulations. 


\subsection{Case Description}

The synoptic conditions have a large effect on the CBL turbulence, and we restricted our study to almost cloud-free days with little large-scale advection. In the presence of clouds, the incoming radiation at the surface can show a high spatial variability that is superimposed upon the surface heterogeneity, while an overcast sky eliminates the influence of the land-use classes to a great extent. The effect of advection by the background flow, on the one hand, "smears" the heterogeneity signal (this will be discussed in detail in Sect. 3.4). On the other hand, it is very difficult to account for temperature and humidity trends due to larger-scale advection within the simulation. A weak background flow is therefore preferred. An overview of the conditions during the LITFASS-2003 measurement period can be found in Beyrich and Mengelkamp (2006). Four days, May 30, June 2, June 13 and June 17, were selected according to the restrictions mentioned above, with synoptic conditions for all days dominated by anticyclonic conditions. The selected days had minimum/maximum temperatures between 13 and $29^{\circ} \mathrm{C}$. We simulated the diurnal cycle from the early morning until the evening transition.

May 30 was characterized by clear skies and a geostrophic wind from the east at $2 \mathrm{~m} \mathrm{~s}^{-1}$ (hereafter also called the weak wind case, LIT2E). Figure 2a, b shows the prescribed areaaveraged turbulent surface fluxes of sensible and latent heat ( $H$ and $L E$, respectively) in the course of the day. It is obvious that the Bowen ratio $\left(B_{0}=H / L E\right)$ for case LIT2E was between 3 and 4 during daytime. The sensible heat flux increased in the course of the day up to $380 \mathrm{~W} \mathrm{~m}^{-2}$, while the latent heat flux was relatively small $\left(\approx 100 \mathrm{~W} \mathrm{~m}^{-2}\right)$ and only showed a weakly-developed diurnal cycle. Figure $2 \mathrm{c}$, d shows the measured fluxes for the specific land-use classes for case LIT2E and reveals that the forest was the dominant land-use class regarding the heat input into the atmosphere (up to $500 \mathrm{~W} \mathrm{~m}^{-2}$ ). The water patches show no significant contribution to the sensible heat input and rather tended to take up energy in the late afternoon. Areas tilled with triticale and barley exhibited maximum values of $H=300 \mathrm{~W} \mathrm{~m}^{-2}$, while for grass, maize and rape, $H$ reached $180-230 \mathrm{~W} \mathrm{~m}^{-2}$. The largest sensible heat-flux differences were observed between water and forest, while the tilled areas differed only slightly from each other. In contrast to the sensible heat flux, the latent heat flux was characterized by high fluctuations during the course of day with amplitudes up to $50 \%$ of the total flux (e.g. forest areas), and mainly due to by water vapour release events from the plants. The maximum values were within the range of $100-220 \mathrm{~W} \mathrm{~m}^{-2}$. Summed up, the forest patches exhibited the smallest latent heat flux into the atmosphere, due to a low availability of water vapour during a dry period of several weeks ahead of the LITFASS-2003 experiment, while rape was associated with the highest input. An extension of all four lateral boundaries of the model with buffer zones of $10 \mathrm{~km}$ was used for case LIT2E, see Appendix B.

June 2 and June 17 (LIT4SE and LIT3SE) displayed higher geostrophic wind speeds of 4 and $3.2 \mathrm{~m} \mathrm{~s}^{-1}$, respectively, and consequently, a buffer zone of $14 \mathrm{~km}$ was used. The wind direction was similar (south-east) in both cases and the surface fluxes were comparable to those in case LIT2E (see Fig. 2a, b). The main difference between the two cases was a greater boundary-layer depth in the simulations $(3,050$ and 2,400 $\mathrm{m}$ for cases LIT4SE and LIT3SE, respectively) compared to LIT2E $(2,200 \mathrm{~m})$. Scattered cumulus and cirrus clouds were observed in the morning.

Due to intense precipitation on the previous days, the Bowen ratio on June 13 (LIT6NW) was approximately one; Beyrich et al. (2006) give a detailed description of the Bowen ratio values during the experiment. For LIT6NW a geostrophic wind speed of $6 \mathrm{~m} \mathrm{~s}^{-1}$ was prescribed from the north-west. Due to the effect of advection, a buffer zone of $18 \mathrm{~km}$ was 
required. The overcast sky at 0500 UTC rapidly changed to only scattered cumulus and stratocumulus clouds between 0700 and 0900 UTC.

Table 1 sums up model set-up properties, such as the prescribed geostrophic wind and model domain size, as well as the boundary-layer height as found during the simulations. Homogeneous control runs with a spatially-averaged but temporally-varying surface heat flux according to Fig. 2a, b were carried out for comparison.

\section{Simulation Results}

\subsection{Mean Profiles}

The temporal development of horizontally-averaged vertical boundary-layer profiles for May 30 (LIT2E) and June 13 (LIT6NW) is shown and compared with radiosonde data in Fig. 3. The solid black lines reflect the initial profiles used in the LES. The initial potential temperature profile on May 30 is characterized by a near-surface inversion with a near-neutral stratified residual layer and a capping inversion above (starting from 1,200 m). June 13 exhibits two residual layers and displays a capping inversion at $2,100 \mathrm{~m}$. This results also in a higher CBL depth during the simulations (see Table 1). During daytime, differences in temperature and humidity between radiosonde and the LES are found, while the height of the capping inversion agrees very well. Differences may generally be attributed to the fact that domainaveraged LES profiles are compared with local observations from the radiosonde. In case LIT2E the mixed-layer temperature is overestimated by the LES by $1.5 \mathrm{~K}$ at $1100 \mathrm{UTC}$, while at 1400 UTC it is slightly underestimated by $0.6 \mathrm{~K}$. At the end of the simulation (1700 UTC) both observations and LES compare very well. The humidity profiles for LIT2E show that the LES slightly underestimated the moisture in the CBL starting from 1400 UTC. However, the radiosonde data show a rather fluctuating humidity in the course of the day and generally they compare well with the LES. In case LIT6NW we had to adjust the initial profiles of temperature and humidity since clouds were observed in the early morning hours that are clearly visible in the humidity profile starting from $900 \mathrm{~m}$ and which we could not account for in the LES. The initial LES profiles were thus defined in such a way that they agree with measurements between 0700 and 0800 UTC and at the end of the simulation. Nevertheless it appears that, as in case LIT2E, differences in the temperature up to $1.5 \mathrm{~K}$ are present, while the CBL height fits well. The humidity profiles during daytime differ by up to $1.5 \mathrm{~g} \mathrm{~kg}^{-1}$ between LES and the radiosonde measurements.

In spite of the local character of the radiosonde profiles, the temporal development and shapes of the simulated mean vertical profiles of potential temperature and specific humidity $(q)$ as well as $\left\langle z_{i}\right\rangle$ in the course of the day compare well with observations. For LIT3SE and LIT4SE there is an even better agreement (not shown). Hence, using the representative surface-flux measurements for the different land-use classes as the surface boundary condition seems to provide an appropriate forcing for the simulations. This is a prerequisite for any further analysis of the simulation data.

Figure 4 shows vertical profiles of the scalar fluxes at 1300 UTC for the LITFASS simulations and the homogeneous control runs. Both the kinematic heat flux and moisture flux display classical profiles (see e.g. Deardorff 1974; Wyngaard and Coté 1974; Stull 1988). The entrainment flux minimum is about $20 \%$ of the normalized surface flux. The humidity in the boundary layer is rather dominated by the mixing of dry air from the free atmosphere into the boundary layer. Hence, the moisture flux in the entrainment layer is two to three times larger than the respective surface flux. We return to the scalar flux profiles later in Sect. 3.3. 


\subsection{Secondary Circulations During LITFASS-2003}

\subsubsection{Spatial Characteristics}

The ensemble averaging method (Eq. 1) allows us to determine the secondary circulation patterns over complex surface heterogeneities for the first time. The heterogeneity-induced vertical velocity $w_{\text {hi }}$ is an appropriate measure for secondary circulations, since updrafts and downdrafts span the entire boundary layer, reaching maximum values in the middle of the boundary layer. Figure 5 shows isosurfaces of $w_{\text {hi }}= \pm 0.3 \mathrm{~m} \mathrm{~s}^{-1}$ at 1300 UTC for all LITFASS simulations. Please note that cases LIT4SE and LIT3SE were found to exhibit similar characteristics owing to the minor differences in the geostrophic wind speed and direction. Hence, we hereafter often discuss results for case LIT3SE only. It is obvious from Fig. 5b, d that the general pattern for both cases is similar. However, the specific locations of updrafts and downdrafts change significantly even for small changes in the geostrophic wind. For case LIT2E, the shown structures are stationary for the period 1000-1600 UTC, while the other cases display a higher variability in time that is discussed for case LIT6NW in Sect. 3.2.2. Most of the secondary circulation structures in case LIT2E are obviously associated with the underlying thermal heterogeneity (see Figs. 1, 5e). Especially the water-forest discontinuities are connected to updraft and downdraft secondary circulation branches, owing to the surface sensible heat-flux gradients discussed in the previous section (see Fig. 2c). This contrast leads to strong secondary circulations that indicate the underlying surface heterogeneity. The weak background flow from the east has only a minor effect on the secondary circulation patterns. Contrary to the forest-water discontinuities, which cause strong circulations, the heterogeneity-induced circulations over the agricultural dominated area are weaker and the influence of agricultural land-use heterogeneity on the CBL can be regarded as small. One reason for this relates to the length scale of the agricultural heterogeneities, which is often less than the boundary-layer depth and thus does not affect the boundary-layer structure (Shen and Leclerc 1995). Overall, the vertical velocities $w_{\text {hi }}$ exhibit typical maximum values of about $15 \%$ of the mixed-layer convective velocity scale $w_{*}$ (after Deardorff 1974) and hence are one order of magnitude smaller than the randomly distributed convective updraft and downdrafts. The secondary circulations are just a small superposition on the primary circulation.

The background flow has a stronger influence on the other three cases in such a way that direct correlations between the secondary circulations and the surface heterogeneity are not obvious from Fig. 5b-d, but we show in Sect. 3.4 that correlations also persist for these cases with higher background wind speeds of $4-6 \mathrm{~m} \mathrm{~s}^{-1}$. Cases LIT4SE and LIT3SE show rolllike structures. Case LIT6NW shows a distinct development of such long-ranged rolls all day long, oriented parallel to the mean wind direction (Fig. 5c). The secondary circulation in case LIT6NW appears as a more slender roll-like pattern. Only few structures develop, indicating that the circulation strength is significantly decreased compared to the weaker wind cases (to be discussed below). This is in agreement with the studies of Avissar and Schmidt (1998) and RH01 who noted a damping effect of the background flow on the surface heterogeneity signal. Both studies identified the formation of rolls over idealized heterogeneities. Moreover, RH01 showed that, for two-dimensional heterogeneities, the formation of rolls depends on the flow direction relative to the orientation of the heterogeneity. The present study shows for the first time that roll-like patterns also develop over irregular heterogeneities and that they seem to be a frequent feature for situations with higher background wind speeds.

The vertical profiles of the variance of the heterogeneity-induced velocity components $\sigma_{u_{\mathrm{hi}}}^{2}, \sigma_{v_{\mathrm{hi}}}^{2}$ and $\sigma_{w_{\mathrm{hi}}}^{2}$ as well as of heterogeneity-induced temperature and humidity variations 


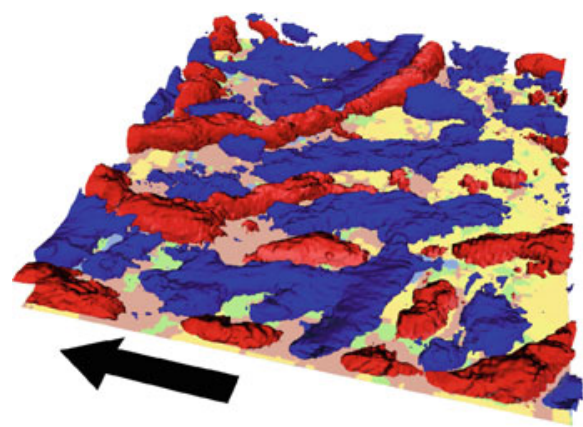

(a) LIT2E

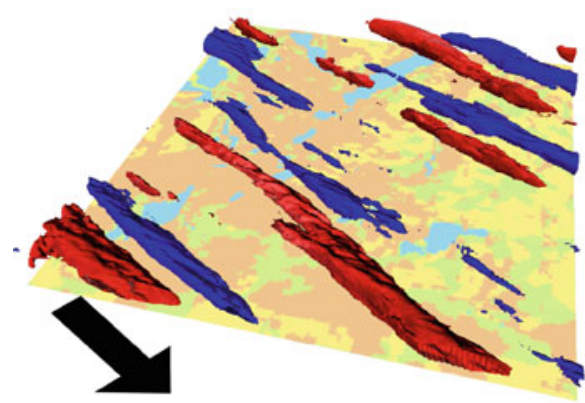

(c) LIT6NW

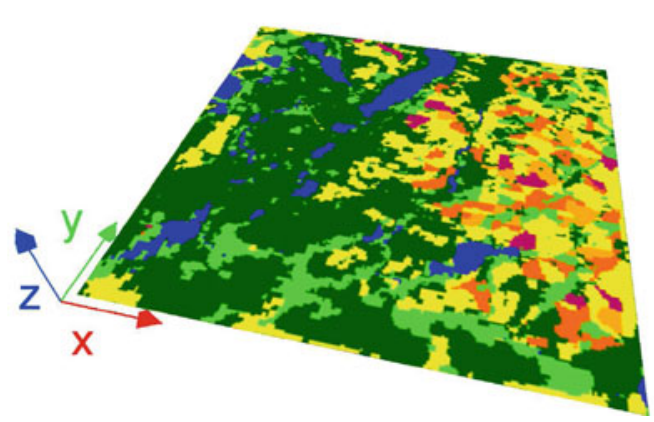

(e) Distribution of land-use classes in $A_{\mathrm{L}}$

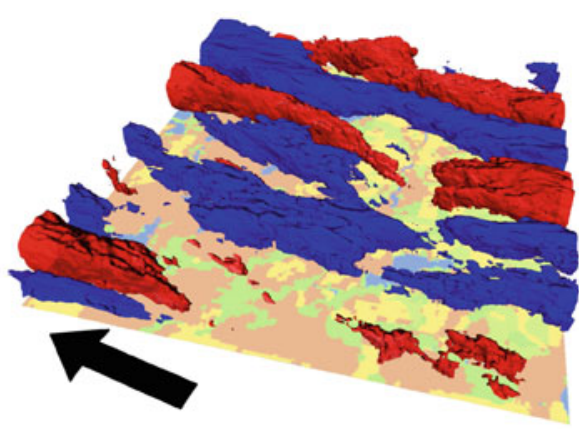

(b) LIT4SE

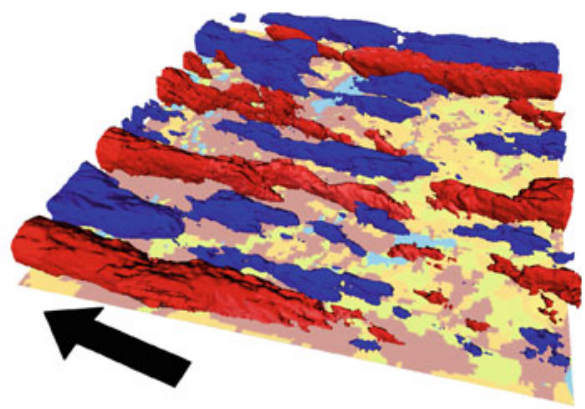

(d) LIT3SE

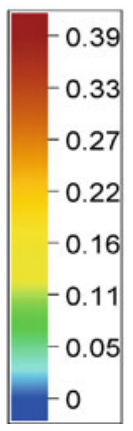

Fig. 5 Secondary circulations over $A_{\mathrm{L}}$ (the area shown is $20 \mathrm{~km} \times 20 \mathrm{~km}$ ), determined from the LITFASS simulations at 1300 UTC (a-d). The figure shows isosurfaces of the heterogeneity-induced vertical velocity. The red isosurface refers to $w_{\mathrm{hi}}=0.3 \mathrm{~m} \mathrm{~s}^{-1}$ (updrafts), the blue one to $w_{\mathrm{hi}}=-0.3 \mathrm{~m} \mathrm{~s}^{-1}$ (downdrafts). The bottom surface displays the prescribed kinematic heat flux according to the legend (in $\mathrm{K} \mathrm{m} \mathrm{s}^{-1}$ ). e Land-use classes as in Fig. 1 and the orientation to the numerical grid. The prescribed geostrophic wind direction is indicated by the arrows (eye-fitted, see Table 1)

( $\sigma_{\theta_{\mathrm{hi}}}^{2}$ and $\sigma_{q_{\mathrm{hi}}}^{2}$, respectively) for the four cases are shown in Fig. 6 . The variance profiles of the heterogeneity-induced temperature and humidity variations (Fig. 6c-e) show the same characteristics as for the primary circulation in a CBL (see e.g. Stull 1988). The variance $\sigma_{w_{\text {hi }}}^{2}$ increases with height to peak values in the middle of the CBL, while the peak magnitude of $\sigma_{w_{\mathrm{hi}}}^{2}$ decreases from case LIT2E $\left(0.26 \mathrm{~m}^{2} \mathrm{~s}^{-2}\right)$ to LIT6NW $\left(0.06 \mathrm{~m}^{2} \mathrm{~s}^{-2}\right)$, reflecting the 


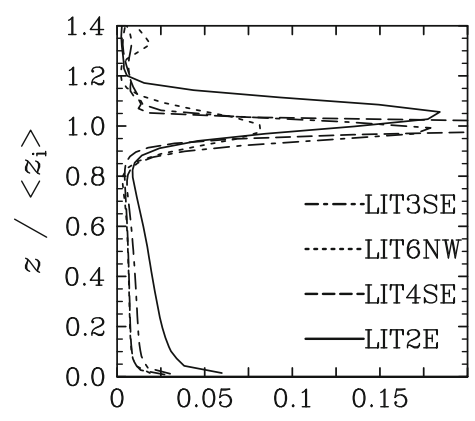

(a) $\sigma_{\theta_{h i}}^{2}\left(K^{2}\right)$

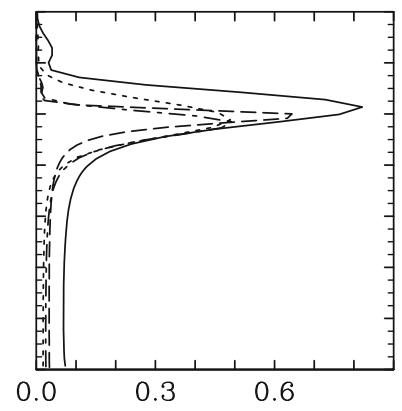

(b) $\sigma_{q_{h i}}^{2}\left(g^{2} \mathrm{~kg}^{-2}\right)$
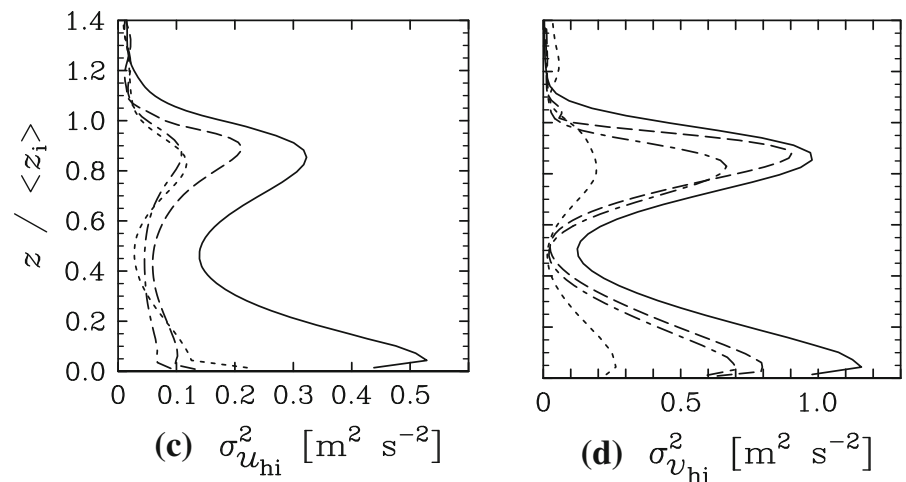

(d) $\sigma_{v_{\mathrm{hi}}}^{2}\left[\mathrm{~m}^{2} \mathrm{~s}^{-2}\right]$

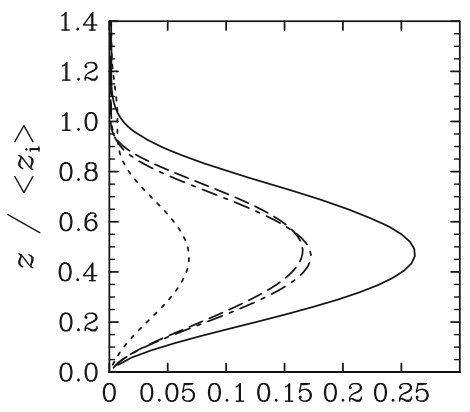

(e) $\sigma_{w_{h i}}^{2}\left[m^{2} s^{-2}\right]$

Fig. 6 Profiles of the variances of the heterogeneity-induced potential temperature and specific humidity variations $(\mathbf{a}, \mathbf{b})$ as well as velocity components (c-e) at 1300 UTC. The height is normalized with $\left\langle z_{i}\right\rangle$

relative strength of secondary circulations very well. The profiles of $\sigma_{u_{\mathrm{hi}}}^{2}$ and $\sigma_{v_{\mathrm{hi}}}^{2}\left(u_{\mathrm{hi}}\right.$ and $v_{\text {hi }}$ refer to the velocity in the $x$ - and $y$-directions, respectively) both exhibit double peaks, a lower one close to the surface and an upper one near the top of the CBL. These peaks are connected to the horizontal secondary circulation branches, which typically occur near the top and the bottom of the CBL, showing that the secondary circulations span the entire boundary layer. Similar-shaped profiles have been shown by PSM05. The variances of the horizontal velocity components $\sigma_{u_{\mathrm{hi}}}^{2}$ and $\sigma_{v_{\mathrm{hi}}}^{2}$ also clearly show that secondary circulations 


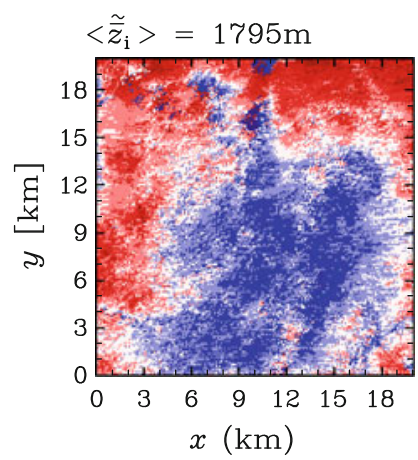

(a)

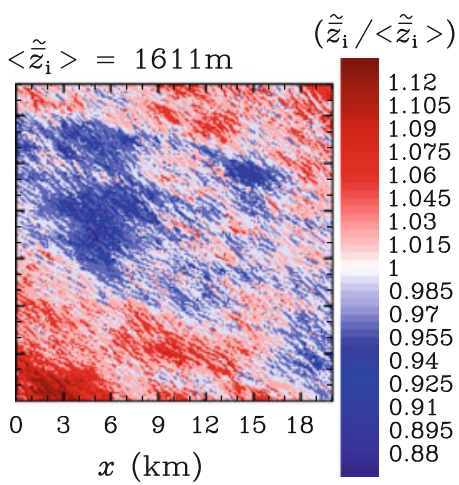

(b)

Fig. 7 Horizontal cross-sections of the ensemble- and time-averaged $z_{i}$ at 1300 UTC over $A_{\mathrm{L}}$ for cases LIT2E (a) and LIT6NW (b). The values are normalized by the horizontal mean $\left\langle\widetilde{\overline{z_{i}}}\right\rangle$ (noted down just above the plots)

become weaker with increasing background wind speed. Our study shows that this effect of the background wind speed on the secondary circulation strength, which was found in several idealized studies before (e.g. Hadfield et al. 1992; Avissar and Schmidt 1998), also holds for complex surface heterogeneities.

In contrast to $\sigma_{w_{\mathrm{hi}}}^{2}, \sigma_{\theta_{\mathrm{hi}}}^{2}$ and $\sigma_{q_{\mathrm{hi}}}^{2}$ exhibit high maxima at the top of the CBL (see Fig. 6a, b), caused by a folding of the interface between the boundary layer and free atmosphere. The high variances of the heterogeneity-induced temperature and humidity variations are a result of the variations in the local boundary-layer height. The time- and ensemble-averaged $z_{i}$ is shown in Fig. 7. In case LIT2E (see Fig. 7a) the large forest patches to the west and the north of $A_{\mathrm{L}}$ (high surface sensible heat flux), lead to an increase in the boundary-layer depth of up to $13 \%$, while agricultural fields and lakes generally exhibit a lower $\widetilde{\overline{z_{i}}}$. This folding of the inversion layer was also shown by HA08, and it is probably caused by the higher input of sensible heat over warmer surface patches, particularly forest areas, that increases the local boundary-layer temperature and hence $\widetilde{\overline{z_{i}}}$ (encroachment effect, Stull 1988, Chap. 11.2). The

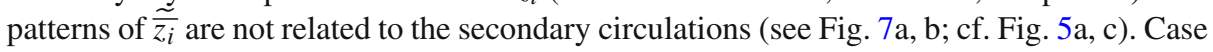
LIT2E shows that secondary circulations at the sharp edges of the heterogeneities (Fig. 5a, e) correlate with the regions of sharp gradients in $\widetilde{\overline{z_{i}}}$, but they do not extend over the centre of larger patches. This finding that secondary circulations develop only at sharp edges of heterogeneities is in agreement with RH01, who have shown this effect for a discontinuous heat-flux amplitude (cf. RH01, Fig. 4). In case LIT6NW (see Fig. 7b) the CBL is deeper in the south-west as well as in the north-east, while lower $\widetilde{z_{i}}$ is found at the centre of $A_{\mathrm{L}}$. In this case it is difficult to relate the pattern to the surface heterogeneity and the encroachment effect because the mean flow "smears" the surface signal significantly.

Near the surface, the variance of the heterogeneity-induced temperature variations (Fig. 6a) shows a lower secondary maximum that is generated by the heterogeneous surface sensible heat flux, while $\sigma_{q_{\mathrm{hi}}}^{2}$ does not show this secondary peak. This proves that the variation in the surface latent heat flux is only of minor importance to the heterogeneity-induced structures.

\subsubsection{Temporal Development}

The secondary circulation patterns broaden in the course of the day; (Fig. 8) e.g. shows this broadening for case LIT6NW during the period from 1000 to 1500 UTC. This can be 


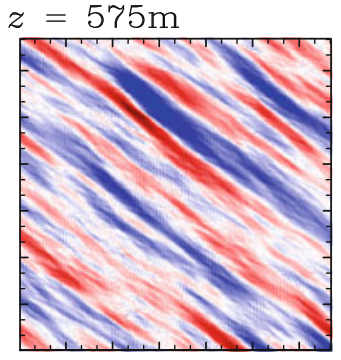

(a) 1000 UTC

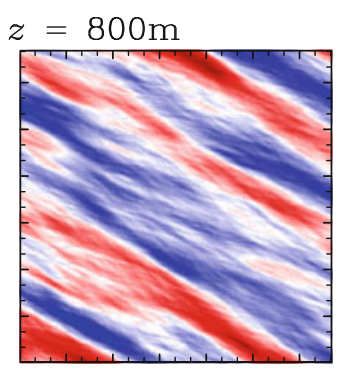

(b) 1300 UTC

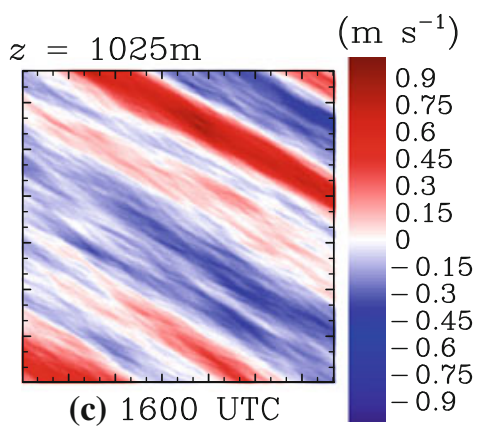

Fig. 8 Horizontal cross-sections of the vertical velocity $w_{\text {hi }}$ over $A_{\mathrm{L}}$ (the area shown is $20 \mathrm{~km} \times 20 \mathrm{~km}$, axes are oriented as in Fig. 7) at $z /\left\langle z_{i}\right\rangle=0.5$ for different times during LIT6NW. The height level is noted above the plots

explained by the findings of Shen and Leclerc (1995) and RH01 that only those heterogeneities with $\lambda /\left\langle z_{i}\right\rangle \geq 1$ are able to extend throughout the entire CBL. Since the prescribed heterogeneities range over different scales and the boundary-layer height increases in the course of the day (see Fig. 3), the scales that are dominantly affecting the boundary layer also change in time. In this way, smaller scales become less and larger scales more important, resulting in broader secondary circulation patterns. Our results clearly show that the dependency of secondary circulations on $\lambda /\left\langle z_{i}\right\rangle$ is also valid for irregularly distributed heterogeneities that cover a large range of scales. Hence the results of Liu et al. (2011), who simulated the flow over two-dimensional heterogeneities with a constant wavelength $\lambda$ over several hours, can be generalized. They showed that the secondary circulations break down after a few hours, since the boundary-layer depth increased such that $\lambda /\left\langle z_{i}\right\rangle$ becomes too small. Due to the different scales of the LITFASS heterogeneity, such a break-down does not occur, and instead the circulation scale increases in time (broadening, see Fig. 8).

\subsection{The Impact of Secondary Circulations on the Area-Averaged Vertical Transport}

In order to determine the area-averaged flux $\langle\overline{w \Phi}\rangle$, we decomposed the total flux into heterogeneity-induced and small-scale turbulent parts according to Eq. 3. In the homogeneouslyheated CBL the heterogeneity-induced part is zero and the total flux equals the turbulent flux.

Results are provided for 1300 UTC. Figure 4 shows the mean profiles of the total fluxes $\langle\overline{w \theta}\rangle$ and $\langle\overline{w q}\rangle$, which were discussed in Sect. 3.1. It also includes the heterogeneity-induced and turbulent contributions to the mean flux as well as the flux from the respective homogeneous control runs. The heterogeneity-induced part $\langle w \theta\rangle_{\mathrm{hi}}$ has its maximum at $z /\left\langle z_{i}\right\rangle=0.3$ and an entrainment minimum at $z /\left\langle z_{i}\right\rangle=0.9$. The maximum value depends on the secondary circulation strength (strongest for case LIT2E, weakest for case LIT6NW) and varies between $20 \%$ (LIT2E) and $8 \%$ (LIT6NW) of the total flux at the corresponding height. In general, the turbulent part $\langle w \theta\rangle_{\mathrm{S}}$ makes up the bulk of the total flux. These qualitative results are in agreement with the LES studies of PSM05 and HA08. Compared to the homogeneous control runs, the absolute total fluxes in the entrainment zone are smaller in the presence of surface heterogeneities. Figure 4 shows that the entrainment rate depends on the secondary circulation strength in such a way that stronger secondary circulations decrease the entrainment fluxes. Because Lilly (2002) has shown that the horizontal averaging process for the turbulent flux profiles may cause misleading results in the case of heterogeneously driven boundary 
layers, we also analyzed the time series of $\left\langle z_{i}\right\rangle$, derived from the local temperature profiles (Eq. 4). This methods confirms a slower growth of $\left\langle z_{i}\right\rangle$ in the heterogeneous cases with $\left\langle z_{i}\right\rangle$ about 50-100 m lower compared to the homogeneous control runs (not shown). This result is in contrast with results from HA08, who did not find any effect of one-dimensional idealized heterogeneities on the entrainment rate. We suppose that at the top of the mixed layer the secondary circulations re-direct a part of the total vertical transport to horizontal transport that decreases the entrainment rate. We are currently analyzing the entrainment modification in detail and will address it in a follow-up paper.

Our main result is that, under the same mean surface fluxes, the total vertical transport above homogeneous and heterogeneous surfaces differs slightly in the entrainment zone. Compared with the homogeneous case, the turbulent transport in the heterogeneous case is smaller in the mixed layer, but secondary circulations compensate for the difference.

As with $\langle w \theta\rangle_{\mathrm{hi}},\langle w q\rangle_{\mathrm{hi}}$ depends on the secondary circulation strength, with its maximum varying between $39 \%$ (LIT2E) and $11 \%$ (LIT6NW) of the total flux. The findings of the present study suggest a smaller heterogeneity-induced part compared to PSM05, who found the heterogeneity-induced part contributes the bulk of the total flux under certain conditions. Unlike $\langle\overline{w \theta}\rangle,\langle\overline{w q}\rangle$ can deviate significantly from the homogeneous control runs (Fig. 4e, f). The maximum is located at $z /\left\langle z_{i}\right\rangle=0.8$. It is decreased by $2 \%$ in case LIT2E, compared to the homogeneous control run, while in case LIT6NW, $\langle\overline{w q}\rangle$ is increased up to $18 \%$. The latter is surprising, since the total flux profile is rather expected to approach the homogeneous control run profile, if the secondary circulation weakens. As the synoptic conditions differ from case to case, the behaviour of the moisture flux is probably caused by different inversion strengths and humidity levels in the free atmosphere (see e.g. Fig. 3). However, we could not clearly identify the reasons for the behaviour of $\langle\overline{w q}\rangle$. The quite large differences between homogeneous and heterogeneous simulations of up to $18 \%$ point out that the effect of surface heterogeneities might be relevant for the parametrization of turbulence in numerical weather prediction models that cannot resolve secondary circulations in the range of a few kilometres.

In contrast to former idealized studies (e.g. Chen and Avissar 1994; Lynn et al. 1995; PSM05), who found that the secondary circulations can contribute up to 50-80\%, our investigations of the LITFASS area show a contribution up to $20 \%$ (kinematic heat) and $39 \%$ (moisture).

\subsection{Correlations Between the Secondary Circulations and the Surface Heterogeneity}

From Fig. 5 it is evident that for all cases with higher wind speed (LIT4SE, LIT3SE, LIT6NW) the secondary circulation structure cannot be easily related to the surface heat-flux pattern, i.e. there seems to be no simple correlation between them. It is also evident that for these cases the secondary circulation patterns switch to more simple roll-like patterns, compared with the pattern of case LIT2E (Fig. 5a). In order to explain the location and strength of the roll-like circulations, our starting hypothesis was that in cases with higher mean wind speed the air advected over the heterogeneities "feels" only a mean surface heat flux that is the surface heat flux averaged along its path. By this "smearing" process, the two-dimensional reduces to a one-dimensional surface heterogeneity that varies only along the crosswind direction. The resulting stripe-like heat-flux pattern should generate roll-like circulations with axes aligned to the mean flow. This would be in agreement with the LES study of RH01, where the development of roll-like secondary circulations was shown under a background wind speed of $7.5 \mathrm{~m} \mathrm{~s}^{-1}$. They showed that, for an appropriate inflow direction with respect to the heterogeneity, rolls can develop owing to a stretching effect of their near-surface checkerboard temperature pattern. 
Table 2 List of sensitivity study cases

\begin{tabular}{llc}
\hline Case & Background wind speed $\left(\mathrm{m} \mathrm{s}^{-1}\right)$ & Buffer zone $(\mathrm{km})$ \\
\hline BUF202 & 2 & 2 \\
BUF206 & 2 & 6 \\
BUF210 & 2 & 10 \\
BUF214 & 2 & 14 \\
BUF218 & 2 & 18 \\
BUF402 & 4 & 2 \\
BUF406 & 4 & 6 \\
BUF410 & 4 & 10 \\
BUF414 & 4 & 14 \\
BUF418 & 4 & 18 \\
BUF606 & 6 & 6 \\
BUF610 & 6 & 10 \\
BUF614 & 6 & 14 \\
BUF618 & 6 & 18 \\
BUF622 & 6 & 22 \\
\hline
\end{tabular}

For proving this hypothesis, we used a correlation analysis, where we calculated the linear correlation between the surface kinematic heat flux and the vertical velocity, both averaged along the direction of the roll-axis. Case LIT6NW was chosen due to the distinct roll-like secondary circulation pattern (see Fig. 5c) under a high background wind speed of $6 \mathrm{~m} \mathrm{~s}^{-1}$. The surface kinematic heat flux and $w_{\text {hi }}$ at a height $z /\left\langle z_{i}\right\rangle=0.5$ were averaged along the entire model domain. However, path-averaging of the surface heat flux in the LITFASS cases is problematic since the flux varies in time because of the diurnal cycle. Therefore we decided in the first instance to use data from the buffer sensitivity simulations where the surface forcing was constant in time (BUF606-BUF622, see Table 2). The cases differ in the buffer zone sizes and thus the domain size varied between $28 \mathrm{~km} \times 32 \mathrm{~km}$ and $44 \mathrm{~km} \times 64 \mathrm{~km}$. In contrast to case LIT6NW, easterly winds were prescribed. Because of their different domain sizes these cases provided different heterogeneity patterns and hence were a suitable set of simulations with different surface properties to test our hypothesis under more idealized conditions.

We decided to perform the streamwise average over a distance of $129.6 \mathrm{~km}$, which is the distance a parcel of air travels on average during the simulation time of $6 \mathrm{~h}$ while it is affected by the underlying heterogeneity. A rotation of the data fields towards the mean wind direction was required and carried out, where the mean wind vector was calculated as the spatial average of the horizontal velocity components at $0.5\left\langle z_{i}\right\rangle$ (height of the maximum of $\left.\sigma_{w_{\mathrm{hi}}}\right)$. The data of the original domain were extended by cyclic repetition along the horizontal directions to allow for averaging over a distance of $129.6 \mathrm{~km}$. The heterogeneity-induced vertical velocity $w_{\text {hi }}$ after $6 \mathrm{~h}$ (60-min average) of simulation time was used for the analysis. Figure 9 shows an example (here case BUF614) of the rotated and extended surface heat flux and the associated vertical velocity at $z /\left\langle z_{i}\right\rangle=0.5$. The rotated axes are denoted by $x^{\prime}$ (alongwind) and $y^{\prime}$ (crosswind), while the sections are oriented along the mean flow, which enters from the right side. Due to the cyclic repetition, the surface heat flux shows repeating structures like lakes, where the lowest values (blue) can be found, or larger forest patches, represented by higher values (red). Consequently, the secondary circulation pattern (Fig. 9a) 

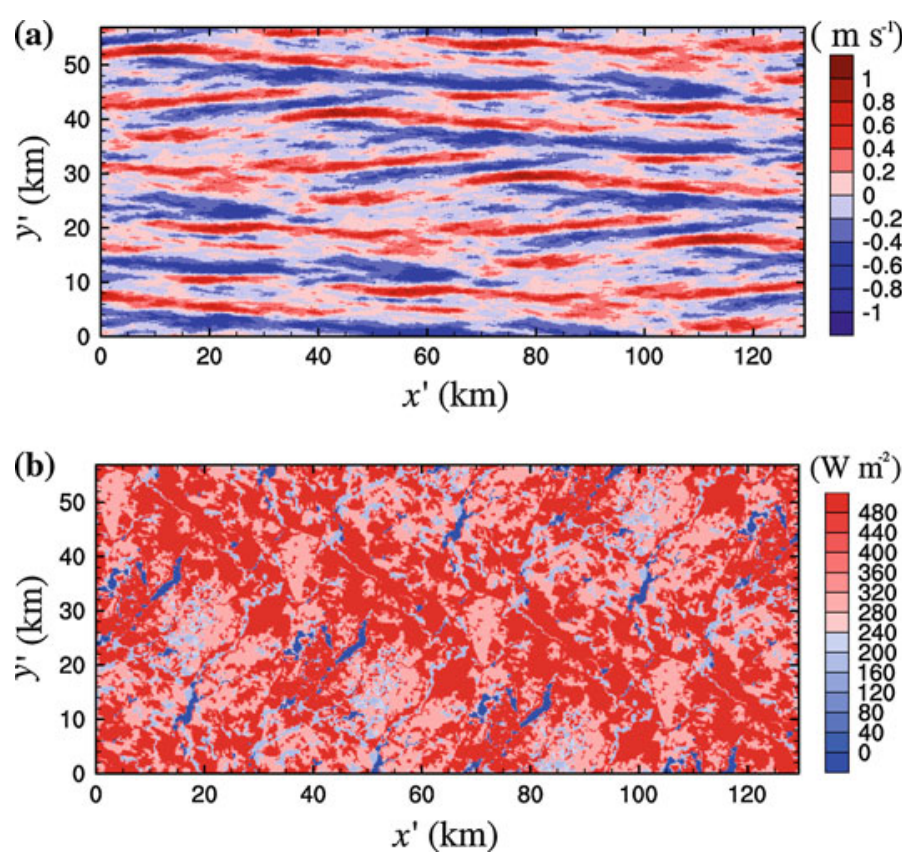

Fig. 9 Horizontal cross-sections of the rotated and extended fields of $w_{\text {hi }}$ at $z /\left\langle z_{i}\right\rangle=0.5$ (a) and surface sensible heat flux (b) for case BUF614



(a) $y^{\prime}(\mathrm{km})$

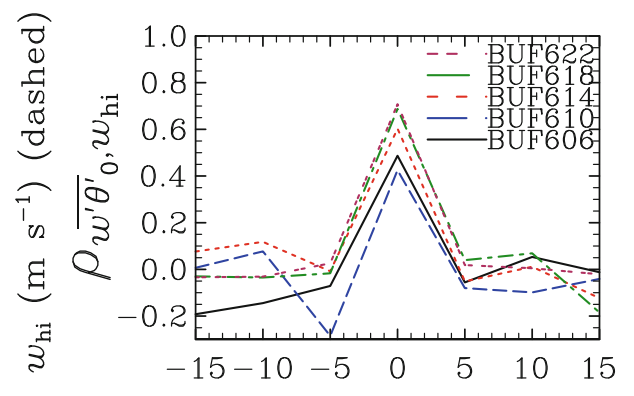

(b) angle (O)

Fig. 10 a Streamwise averages of vertical velocity $w_{\text {hi }}$ at $z /\left\langle z_{i}\right\rangle=0.5$ (blue dashed line) and the surface sensible heat flux $H$ (black solid line) for case BUF614 against crosswind axis $y^{\prime}$, b correlation coefficient between $\overline{w^{\prime} \theta^{\prime}}{ }_{0}$ and $w_{\text {hi }}$ against rotation angle of $\overline{w^{\prime} \theta^{\prime}}{ }_{0}$ in relation to the mean wind

shows repeating structures as well. Anyhow, this wallpaper effect does not affect the general result of the correlation analysis that is valid for any kind of heterogeneity pattern.

\subsubsection{Streamwise-Averaged Correlations}

In order to determine the relation between the secondary circulation structure and the surface heat-flux pattern, we first computed the streamwise average of $w_{\mathrm{hi}}$ at $z /\left\langle z_{i}\right\rangle=0.5$ and $\overline{w^{\prime} \theta^{\prime}} 0$. The crosswind variation of both quantities is given in Fig. 10a (case BUF614) and already displays a good correlation between them. From these one-dimensional series we 
then calculated the correlation coefficient $\varrho_{\overline{w^{\prime} \theta_{0}^{\prime}}, w_{\mathrm{hi}}}$. Additionally we varied the rotation angle of the surface heat-flux field in order to ensure that marginal changes in the rotation angle give totally different streamwise averages that are not correlated to the secondary circulation pattern. Figure 10b shows the correlation coefficient against different angles relative to the mean wind direction. A well-defined peak can be found if the heat flux is streamwise averaged (zero angle) with values of at least 0.4 . A variation of the rotation angle of only $5^{\circ}$ in any direction decreases the correlation to values close to zero and indicates a clear statistical relation between the secondary circulation and the streamwise-averaged surface heat flux.

The correlation coefficient at zero degree rotation increases with increasing area size and reaches a value of 0.7 (Fig. 10b). The same correlation analysis applied to the buffer zone cases with $v_{\mathrm{g}}=4 \mathrm{~m} \mathrm{~s}^{-1}$ (BUF402-BUF418) led to correlations between 0.4 and 0.6. Our hypothesis thus seems to be valid for idealized cases with stationary heat-flux pattern.

Despite the high correlation, systematical shortcomings should be considered that arise from the streamwise average and which are visible e.g. in Fig. 9a. Signals of rolls can be weakened if they do not exactly align with the mean flow or if they do not range over the entire data fields. It is obvious that the secondary circulation at a specific location can only be influenced by the surface heat-flux distribution of a limited upstream region, or in other words, every location has its own footprint (a common issue for turbulence measurements). It is obvious that the size of this footprint will strongly depend on the magnitude of the background wind speed. In Sect. 3.4.2 we will therefore determine the fetch, i.e. the length of this secondary circulation footprint, for different background wind speeds.

We further applied the correlation analysis to case LIT6NW, where the surface heat flux varied with time. To avoid errors in the surface heat flux and the secondary circulation pattern by the diurnal cycle of secondary circulations and surface fluxes, we restricted the averaging distance to the size of the model domain and used the averaged surface heat flux from 1200 to 1300 UTC as well as the secondary circulation at 1300 UTC, which implies a time average of $1 \mathrm{~h}$. The correlation calculated for LIT6NW is also large (0.48), which reveals that our starting hypothesis provides a conclusive explanation for the observed rolls in Fig. 5.

\subsubsection{Fetch Study}

In this section we will extend the correlation analysis by a method to determine the fetch of the secondary circulations. In order to study if and how the fetch depends on the background wind speed, we used the simulated cases from the sensitivity study (listed in Table 2 in Appendix B) with background wind speeds between 2 and $6 \mathrm{~m} \mathrm{~s}^{-1}$.

Instead of using streamwise averages ranging over the entire data fields, we now calculated the correlation between the local vertical velocity $w_{\text {hi }}\left(y^{\prime}\right)$ at $z /\left\langle z_{i}\right\rangle=0.5$ at a specific alongwind location $x_{i}^{\prime}$, where $y^{\prime}$ is the crosswind direction, and the surface kinematic heat flux, streamwise averaged in the upwind direction between $x_{i}^{\prime}$ and $x_{i}^{\prime}+\Delta_{\mathrm{f}} x^{\prime}$. Here, $\Delta_{\mathrm{f}} x^{\prime}$ is a length that varied between 1 and $100 \mathrm{~km}$ (see also Fig. 11). The correlation coefficient $\varrho_{\overline{w^{\prime} \theta_{0}^{\prime}}, w_{\mathrm{hi}}}\left(x_{i}^{\prime}, \Delta_{\mathrm{f}} x^{\prime}\right)$ thus depends on both the alongwind location $x_{i}^{\prime}$ and $\Delta_{\mathrm{f}} x^{\prime}$, but not on $y^{\prime}$. An horizontal average over all $x_{i}^{\prime}$ (denoted by \langle\rangle$_{x^{\prime}}$ ) is calculated in order to achieve representative statistics for the entire domain. The averaged correlation reads:

$$
\left\langle\varrho_{\overline{w^{\prime} \theta_{0}^{\prime}}}, w_{\mathrm{hi}}\right\rangle_{x^{\prime}}\left(\Delta_{\mathrm{f}} x^{\prime}\right)=\frac{1}{N_{x^{\prime}}} \sum_{i=0}^{N_{x^{\prime}}} \varrho_{\overline{w^{\prime} \theta_{0}^{\prime}}, w_{\mathrm{hi}}}\left(x_{i}^{\prime}, \Delta_{\mathrm{f}} x^{\prime}\right), \quad \forall \Delta_{\mathrm{f}} x^{\prime}=1 \mathrm{~km}, 2 \mathrm{~km}, \ldots, x_{\max }^{\prime},
$$




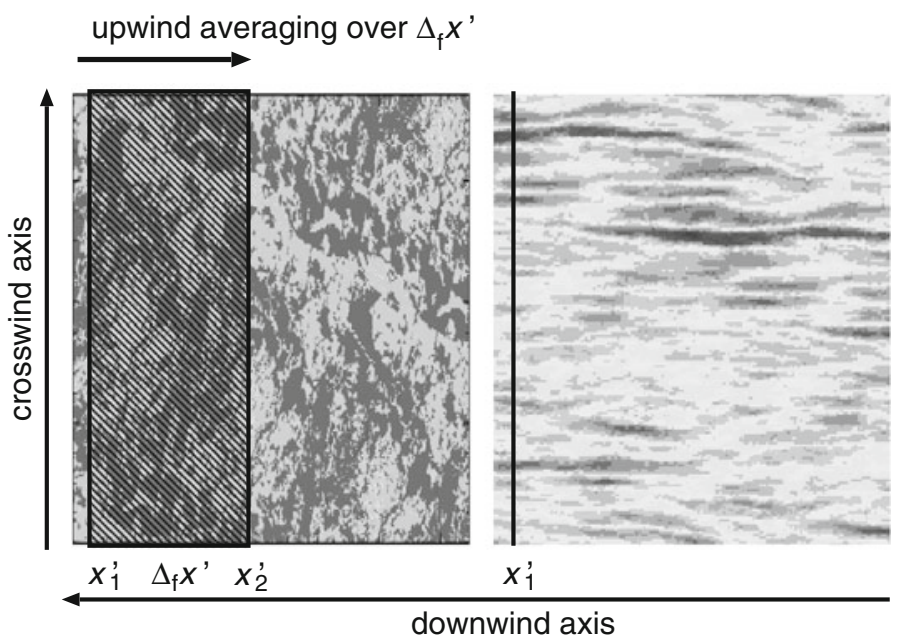

Fig. 11 Schematic figure of the averaging method for the determination of the fetch. The horizontal crosssections of the surface kinematic heat flux (left) and the vertical velocity at $z /\left\langle z_{i}\right\rangle=0.5$ are used. The surface heat flux is streamwise averaged over $\Delta_{\mathrm{f}} x^{\prime}=x_{2}^{\prime}-x_{1}^{\prime}$. The correlation coefficient is calculated from this averaged crosswind surface heat-flux series and the crosswind series of the vertical velocity along the path at $x_{1}^{\prime}$

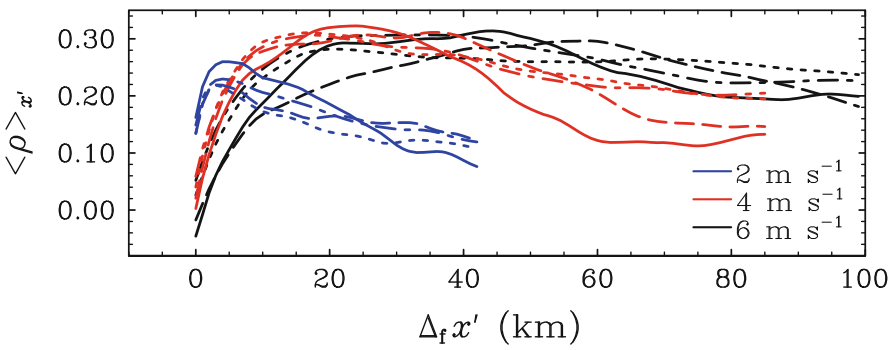

Fig. 12 Spatially-averaged correlation $\left\langle\varrho \overline{w^{\prime} \theta_{0}^{\prime}}, w_{\mathrm{hi}}\right\rangle_{x^{\prime}}$ against averaging length $\Delta_{\mathrm{f}} x^{\prime}$ of the surface heterogeneity. The solid, dot-dashed, short-dashed and dashed lines represent cases with a buffer zone of 6, 10, 14 and $18 \mathrm{~km}$, respectively. The colours mark the wind speed

where $x_{\max }^{\prime}$ varied between 42 and $100 \mathrm{~km}$ depending on the background wind and $N_{x^{\prime}}$ is the number of grid points along the downwind direction.

Figure 12 shows how this correlation depends on $\Delta_{\mathrm{f}} x^{\prime}$. A global maximum of the correlation is found for all cases and it appears that the location of the maximum correlation is similar for equal background wind speeds. For $v_{\mathrm{g}}=2 \mathrm{~m} \mathrm{~s}^{-1}$, the maximum is located at $\left(\Delta_{\mathrm{f}} x^{\prime}\right)_{\max } \approx 5 \mathrm{~km}$, while for higher background winds of $v_{\mathrm{g}}=4 \mathrm{~m} \mathrm{~s}^{-1}$ and $v_{\mathrm{g}}=6 \mathrm{~m} \mathrm{~s}^{-1}$, the maximum shifts to $\left(\Delta_{\mathrm{f}} x^{\prime}\right)_{\max } \approx 19 \mathrm{~km}$ and $\left(\Delta_{\mathrm{f}} x^{\prime}\right)_{\max } \approx 35 \mathrm{~km}$, respectively. Since the correlation more or less monotonically decreases beyond the maximum, we define the position of these maxima as the fetch $\Delta_{\mathrm{f}}$. As expected, it is larger for higher wind speeds. The analysis points towards a linear increase of the fetch with wind speed. It is quite evident that the buffer zone size should ideally cover the fetch in the upstream model boundaries in order to simulate realistic secondary circulation patterns. For our simulations we used buffer 


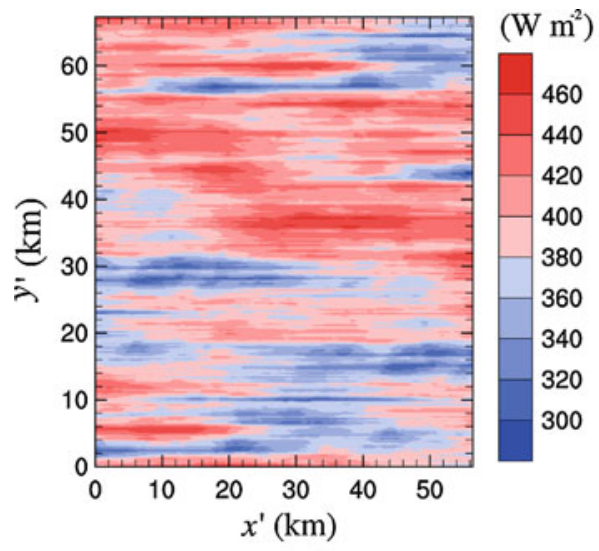

(a) $\langle H\rangle_{\Delta_{\mathrm{f}}}$

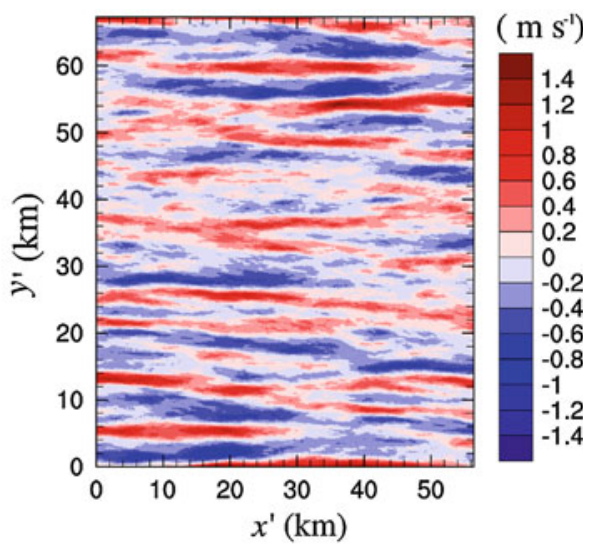

(b) $w_{\mathrm{hi}}$

Fig. 13 Horizontal cross-sections of, a the effective surface sensible heat flux, $\mathbf{b}$ the heterogeneity-induced vertical velocity at $z /\left\langle z_{i}\right\rangle=0.5$ for case BUF618

zones between 10 and $18 \mathrm{~km}$, which should suffice for $v_{\mathrm{g}}=2 \mathrm{~m} \mathrm{~s}^{-1}$ and $v_{\mathrm{g}}=4 \mathrm{~m} \mathrm{~s}^{-1}$. For $v_{\mathrm{g}}=6 \mathrm{~m} \mathrm{~s}^{-1}$, however, buffer zones up to $35 \mathrm{~km}$ might be necessary.

By knowing the fetch, an effective surface sensible heat flux $\langle H\rangle_{\Delta_{\mathrm{f}}}$ can be calculated for each horizontal location in the domain by upstream-averaging of $H$ over the fetch (denoted by \langle\rangle$_{\Delta_{\mathrm{f}}}$ ). These streamwise-averaged surface heat-flux patterns reflect the upstream moving average of the original surface heat-flux pattern. Figure 13 shows $\langle H\rangle_{\Delta_{\mathrm{f}}}$ and $w_{\mathrm{hi}}$ for BUF618. The typical length scale of the $\langle H\rangle_{\Delta_{\mathrm{f}}}$ patches in alongwind direction is in agreement with the respective length of the rolls. Furthermore the crosswind gradients in $\langle H\rangle_{\Delta_{\mathrm{f}}}$ allow us to estimate the development of circulation structures to a high degree (see e.g. the striking rolls in the upper part and the lower left edge of the figure). It thus should be possible to predict the secondary circulation pattern to a considerable extent from a given surface heterogeneity distribution and respective background flow.

\section{Summary and Outlook}

In the present study the CBL over a complex surface heterogeneity, observed during LITFASS-2003, was investigated with the focus on secondary circulations and their impact on the development of the CBL during the daytime. Four cases with varying background wind speeds were simulated from the early morning until the evening transition. The simulations were initialized and driven by surface fluxes and profiles of temperature and humidity observed during LITFASS-2003. The cases were characterized by geostrophic wind speeds between 2 and $6 \mathrm{~m} \mathrm{~s}^{-1}$ and maximum boundary-layer heights between 2,200 and 3,050 m. The mean profiles of temperature and humidity in the course of the simulations showed that the LES model was able to simulate the observed CBL development.

Particular attention was given to the development and three-dimensional structure of the heterogeneity-induced secondary circulations. A new method was introduced in analogy to former studies, based on a combined extensive time- and ensemble-averaging, to separate the heterogeneity-induced secondary circulations from the randomly distributed thermal convection. Furthermore, a sensitivity study showed that, in the case of irregularly distributed 
surface heterogeneities, sufficiently large buffer zones need to be defined around the analysis domain in order to capture effects on secondary circulations from regions further upstream.

The simulated secondary circulations during the four LITFASS simulations spanned the entire CBL and were superimposed on the randomly distributed convection. The circulation strength was found to be typically one order of magnitude smaller than turbulence of the primary circulation. Increasing background wind speeds tended to weaken the secondary circulations as was previously found in idealized studies (e.g. Avissar and Schmidt 1998; RH01). For low wind speeds of $2 \mathrm{~m} \mathrm{~s}^{-1}$ the complex secondary circulation patterns could be directly linked to the underlying sensible heat-flux pattern. For higher wind speeds of $3-6 \mathrm{~m} \mathrm{~s}^{-1}$ roll-like structures were observed. For the latter cases, a correlation analysis proved that the flow "feels" only the mean surface heat-flux patterns that derive from the original patterns by averaging them in the streamwise direction. Because these effective patterns are basically aligned to the direction of the mean flow, they generate roll-like secondary circulation patterns. The patterns are found to be mainly controlled by the upstream surface conditions. The upwind fetch of the surface heterogeneity pattern that controls the secondary circulation was found to depend strongly on the background wind speed. Our results point towards a linear increasing fetch with increasing wind speed. A larger fetch and thus longer effective stripe-like surface heat-flux patches explain the elongated rolls in the simulation with a background wind of $6 \mathrm{~m} \mathrm{~s}^{-1}$. It is shown that the secondary circulation patterns in the simulations can be estimated by calculating the moving average of the surface heat flux in the upstream direction.

The temporal development of the secondary circulations showed that the heterogeneity scales, which affect the CBL, changed in time and smaller scales became less important with increasing $z_{i}$. This resulted in a broadening of the secondary circulations in the course of the day and was associated with the dependency of secondary circulations on the ratio of the heterogeneity scale $\lambda$ to $z_{i}$, which was stated for idealized heterogeneities of a single scale in several studies (e.g. Shen and Leclerc 1995). The present study was able to show that the known dependencies on both $\lambda / z_{i}$ and the background wind speed are also valid over irregular surface heterogeneities, where a whole range of scales are superimposed on each other.

The area-averaged vertical flux of sensible heat suggested that the entrainment of warm air in the case of strong secondary circulations over irregular heterogeneous terrain is slightly reduced compared to the homogeneous control runs. In the mixing layer, the vertical transport was partly taken over by the secondary circulations and the turbulent transport was thus found to be decreased, while the sum of both was not modified. The humidity transport was dominated by drying-out due to high entrainment of dry air from the free atmosphere. The latent heat flux showed different responses to secondary circulations, resulting in either a higher or smaller total flux compared to the homogeneous simulations. The reasons for the behaviour of the latent heat flux remain unclear, and might be the result of different conditions in the free atmosphere, and changes in the entrainment of dry air, or an effect of horizontal averaging.

Our simulations illustrated that the surface heterogeneity affects the boundary layer up to the capping inversion that is also reflected in a horizontally varying boundary-layer depth. The often discussed concept of a blending height, above which the influence of the surface heterogeneity vanishes, thus cannot hold, at least under convective conditions and heterogeneity scales larger than $z_{i}$. The present study already demonstrated that extensive averaging is required to filter out the random noise of the thermal convection. Probably, turbulence measurements from field campaigns do not provide enough data to allow for sufficient averaging. This may be an important reason that experiments over heterogeneous terrain in the 
past did not give any clear evidence of heterogeneity-induced effects. We will address this topic in a follow-up study.

Acknowledgments This research was supported by the German Research Foundation (DFG) under grant RA 617/21-1. All simulations were performed on the SGI Altix ICE at The North-German Supercomputing Alliance (HLRN), Hannover/Berlin. We appreciate the two anonymous reviewers for their constructive and valuable comments that helped to improve the manuscript.

Open Access This article is distributed under the terms of the Creative Commons Attribution License which permits any use, distribution, and reproduction in any medium, provided the original author(s) and the source are credited.

\section{Appendices}

\section{Appendix A: Determination of Heterogeneity-Induced Secondary Circulations over Irregular Surface Heterogeneity}

In order to isolate the effect of surface heterogeneities, our starting point is the decomposition procedure described by PSM05. Adapted for irregular heterogeneities this decomposition reads

$$
\Phi(x, y, z, t)=\langle\Phi\rangle(z, t)+\Phi_{\mathrm{hi}}(x, y, z, t)+\Phi_{\mathrm{S}}(x, y, z, t),
$$

where $\langle\Phi\rangle$ is the horizontal mean (global part) of $\Phi$, and $\Phi_{\mathrm{hi}}$ the heterogeneity-induced part. $\Phi_{\mathrm{S}}$ is the so-called background turbulence that includes the smaller-scale resolved and subgrid-scale contributions due to the randomly distributed thermally driven primary circulations. The only way to eliminate the background turbulence from Eq. 6 is to apply an ensemble average (denoted by a tilde). This requires performing an ensemble of LESs, where each simulation uses different initial random perturbations to trigger convection. In this way, the spatial and temporal distributions of background turbulence are completely different in each ensemble run, while the heterogeneity-induced motion remains the same. If the number of ensembles $N$ is large enough, $\widetilde{\Phi}_{\mathrm{s}}$ will tend to zero. Then, $\Phi_{\mathrm{hi}}$ can be calculated from the ensemble-averaged Eq. 6, viz.

$$
\Phi_{\mathrm{hi}}(x, y, z, t)=\widetilde{\Phi}(x, y, z, t)-\widetilde{\langle\Phi\rangle}(z, t) .
$$

However, the number of ensemble runs, which is required to keep the background turbulence signal sufficiently small, was found to be much larger than our computational resources allowed. Therefore, we decided to carry out an additional time average with fixed, non-overlapping time intervals (denoted by the overbar) for each ensemble run, before applying the ensemble average. Time and ensemble averaging of Eq. 6 yields

$$
\bar{\Phi}_{\mathrm{hi}}(x, y, z, t)=\widetilde{\bar{\Phi}}(x, y, z, t)-\widetilde{\overline{\langle\Phi\rangle}}(z, t) .
$$

In order to reduce the background turbulence signal sufficiently, the averaging interval should be at least in the order of a few convective turnaround times $t_{*}=\left\langle z_{i}\right\rangle / w_{*}$ (after Deardorff 1974). On the other hand, the interval should not be too large, because the time average will also affect the heterogeneity induced signal that varies in time. As a compromise we used an interval of $1 \mathrm{~h}$ and calculated averaged quantities for time periods of e.g. 1200-1300 UTC, 1300-1400 UTC, and so forth. For large horizontal domains as used in our simulations, the horizontally- and temporally-averaged quantities do not vary much among the different ensemble runs, i.e. 


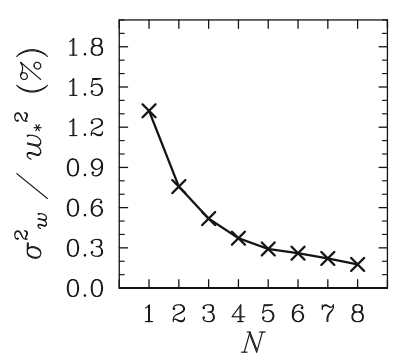

(a)

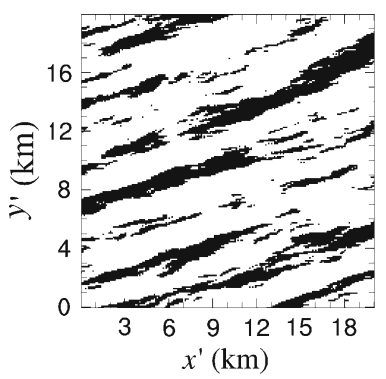

(b)

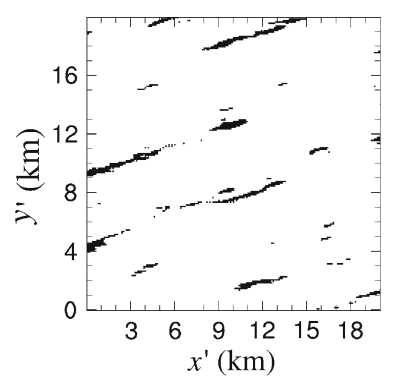

(c)

Fig. 14 Height-, time- and ensemble-averaged and normalized variance of the vertical velocity as a function of the number of ensemble runs $N$ for a homogeneously-heated CBL (a), horizontal cross-sections of the vertical velocity at $z /\left\langle z_{i}\right\rangle=0.5$ for $N=1$ (b) and $N=8(\mathbf{c})$, time averaged over $1 \mathrm{~h}$. Dark shaded regions show areas where $w>0.08 w_{*}$

$$
\widetilde{\overline{\langle\Phi\rangle}} \simeq \overline{\langle\Phi\rangle}
$$

This holds for the first, but also higher order moments, because the mean statistical properties of the flow are the same for all ensemble runs. The $1 \mathrm{~h}$-averaged heterogeneity-induced part $\bar{\Phi}_{\text {hi }}$ can thus be calculated as

$$
\bar{\Phi}_{\mathrm{hi}}(x, y, z, t)=\widetilde{\widetilde{\Phi}}(x, y, z, t)-\langle\bar{\Phi}\rangle(z, t)
$$

Horizontal averaging of Eq. 10 using Eq. 9 yields

$$
\left\langle\bar{\Phi}_{\text {hi }}\right\rangle=0
$$

and hence, $\bar{\Phi}_{\text {hi }}$ can be regarded as a heterogeneity-induced variation from the mean state.

As the number of ensemble runs is practically limited, we carried out a set of sensitivity simulations in order to determine the required amount of ensemble runs to ensure that the random turbulent part $\widetilde{\bar{\Phi}}_{\mathrm{s}}$ is sufficiently small. We used the horizontal variance $\sigma_{\bar{w}_{\text {hi }}}^{2}$, heightaveraged over the entire boundary layer (denoted by $\left.\left(\sigma_{\bar{w}_{\mathrm{hi}}}^{2}\right)_{\mathrm{BL}}\right)$. The sensitivity simulations were carried out for a homogeneously-heated stationary CBL (with $v_{\mathrm{g}}=6 \mathrm{~m} \mathrm{~s}^{-1}$ ), because under such conditions $\bar{w}_{\text {hi }}$ and its variance should tend to zero when sufficiently averaged. As a criterion we defined a limit of $\left(\sigma_{\bar{w}_{\mathrm{hi}}}^{2}\right)_{\mathrm{BL}}$, normalized by the convective velocity scale $w_{*}^{2}$, of $0.2 \%$. Figure 14 a shows how the ratio $\left(\sigma_{\bar{w}_{\mathrm{hi}}}^{2}\right)_{\mathrm{BL}} / w_{*}^{2}$ asymptotically decreases with the number of ensemble runs and the limit is reached for eight ensemble runs. A further reduction of the background turbulence signal would require much more ensemble runs. Figure 14b, c show horizontal cross-sections at $z /\left\langle z_{i}\right\rangle=0.5$ of the 1 -h time-averaged vertical velocity for one of the ensemble runs (Fig. 14b) and the ensemble average over eight runs (Fig. 14c). It is evident that most of the signal from background turbulence, which is still visible in the time-averaged field, has been successfully eliminated for an additional ensemble average over eight runs.

By means of the decomposition of the quantities in Eq. 6, the vertical flux $w \Phi$ can be decomposed as (see also Chen and Avissar 1994):

$$
\begin{aligned}
w \Phi= & \langle w\rangle\langle\Phi\rangle+w_{\mathrm{hi}} \Phi_{\mathrm{hi}}+w_{\mathrm{s}} \Phi_{\mathrm{s}}+\langle w\rangle \Phi_{\mathrm{hi}}+w_{\mathrm{hi}}\langle\Phi\rangle \\
& +\langle w\rangle \Phi_{\mathrm{s}}+w_{\mathrm{s}}\langle\Phi\rangle+w_{\mathrm{hi}} \Phi_{\mathrm{s}}+w_{\mathrm{s}} \Phi_{\mathrm{hi}} .
\end{aligned}
$$


The heterogeneity-induced signal should not vary much within the averaging period of $1 \mathrm{~h}$. We can thus assume that $\overline{\Phi_{\mathrm{hi}}} \approx \Phi_{\mathrm{hi}}$. Since $\Phi_{\mathrm{hi}}$ is also invariant to ensemble-averaging, we can use the relation $\widetilde{\overline{\Phi_{\mathrm{hi}}}} \approx \Phi_{\mathrm{hi}}$. For time and ensemble averaging, the background turbulent part tends to zero, and Eq. 12 then reduces to

$$
\widetilde{\overline{w \Phi}}=\widetilde{\langle w\rangle\langle\Phi\rangle}+w_{\mathrm{hi}} \Phi_{\mathrm{hi}}+\widetilde{\widetilde{w_{\mathrm{s}} \Phi_{\mathrm{s}}}}+\widetilde{\widetilde{\langle w\rangle \Phi_{\mathrm{s}}}}+\widetilde{\widetilde{w_{\mathrm{s}}\langle\Phi\rangle}}+\widetilde{\overline{\langle w\rangle}} \Phi_{\mathrm{hi}}+w_{\mathrm{hi}} \widetilde{\overline{\langle\Phi\rangle}} .
$$

Applying the horizontal average to Eq. 13 yields

$$
\left.\left.\widetilde{\left.\widetilde{w_{\Phi}}\right\rangle}=\widetilde{\langle\overline{\langle w\rangle}\langle\Phi\rangle}+\left\langle w_{\mathrm{hi}} \Phi_{\mathrm{hi}}\right\rangle+\widetilde{\widetilde{\left\langle w_{\mathrm{s}} \Phi_{\mathrm{s}}\right.}}\right\rangle+\widetilde{\widetilde{\langle w\rangle \Phi_{\mathrm{s}}}}\right\rangle+\widetilde{\left.\widetilde{w_{\mathrm{s}}\langle\Phi\rangle}\right\rangle},
$$

noting that the last two terms of Eq. 13 cancel (follows from Eq. 11). As stated above, Eq. 9 holds also for higher order moments. Eq. 14 thus simplifies to

$$
\langle\overline{w \Phi}\rangle=\overline{\langle w\rangle\langle\Phi\rangle}+\left\langle w_{\mathrm{hi}} \Phi_{\mathrm{hi}}\right\rangle+\left\langle\overline{w_{\mathrm{s}} \Phi_{\mathrm{s}}}\right\rangle+\left\langle\overline{\langle w\rangle \Phi_{\mathrm{s}}}\right\rangle+\left\langle\overline{w_{\mathrm{s}}\langle\Phi\rangle}\right\rangle
$$

The inter-scale terms on the right side cancel when substituting the small-scale part according to Eq. 6 and applying common averaging rules. Hence, Eq. 15 reduces to

$$
\langle\overline{w \Phi}\rangle(z)=\overline{\langle w\rangle\langle\Phi\rangle}(z)+\left\langle w_{\mathrm{hi}} \Phi_{\mathrm{hi}}\right\rangle(z)+\left\langle\overline{w_{\mathrm{s}} \Phi_{\mathrm{s}}}\right\rangle(z)
$$

\section{Appendix B: Determination of a Sufficient Buffer Zone Size}

In Sect. 2.2 we explained that irregular surface heterogeneities and cyclic horizontal model boundaries require so-called buffer zones around the analysis area (i.e. the LITFASS domain of $20 \mathrm{~km} \times 20 \mathrm{~km}$, see Fig. 1). If the buffer zones are large enough, secondary circulations in the analysis area should not feel the effects of the cyclic boundaries anymore. We determined the required size of the buffer zones by performing a set of simulations where the buffer size was successively enlarged from 2 to $22 \mathrm{~km}$. Since a strong dependency on the background flow was expected, we also varied the background wind speed between 2 and $6 \mathrm{~m} \mathrm{~s}^{-1}$, flow from the east. We used constant (in time) surface fluxes from the LITFASS experiment on May 30 (1230-1300 UTC) instead of diurnal cycles for two reasons. One reason is that constant fluxes generate steady-state secondary circulation structures within a short time (compared with the time needed for runs where the diurnal cycle was simulated). For the same reason, the initial profile of potential temperature was set with a slightly stable gradient and capping inversion above $1,200 \mathrm{~m}$. The second reason is that the secondary circulation patterns exhibit a diurnal cycle themselves, if the flow is driven by non-stationary surface fluxes. To derive the pure effects of the buffer zone on the secondary circulations would be more difficult under such conditions. An overview of the set of simulations is given in Table 2. The buffer zones for a given background wind speed were considered to be sufficient when the secondary circulation pattern in the analysis area did not change significantly with increasing buffer zone anymore. We explicitly compared the secondary circulation patterns after 6-h simulation time, but we also calculated the change in the vertical velocity between two simulations with different buffer zone size (not shown). Based on this analysis we decided to use a buffer zone of $10 \mathrm{~km}$ for case LIT2E, $14 \mathrm{~km}$ for cases LIT3SE and LIT4SE, and $18 \mathrm{~km}$ for case LIT6NW. Unfortunately we did not find a suitable measure or limiting value for a sufficient buffer zone size, but as discussed in Sect. 3.4.2, the fetch study supports our decision. 


\section{References}

Avissar R, Schmidt T (1998) An evaluation of the scale at which ground-surface heat flux patchiness affects the convective boundary layer using large-eddy simulations. J Atmos Sci 55:2666-2689

Beare RJ, Cortes MAJ et al (2007) An intercomparison of large-eddy simulations of the stable boundary-layer. Boundary-Layer Meteorol 118:247-272

Beyrich F, Mengelkamp HT (2006) Evaporation over a heterogeneous land surface: EVA_GRIPS and the LITFASS-2003 experiment: an overview. Boundary-Layer Meteorol 121:5-32

Beyrich F, Herzog HJ, Neisser J (2002a) The LITFASS project of the DWD and the LITFASS-98 experiment: the project strategy and the experimental setup. Theor Appl Climatol 73:3-18

Beyrich F, Richter SH et al (2002b) Experimental determination of turbulent fluxes over heterogeneous LITFASS area: selected results from the LITFASS-98 experiment. Theor Appl Climatol 73:19-34

Beyrich F, Leps JP et al (2006) Area-averaged surface fluxes over the LITFASS region based on eddy-covariance measurements. Boundary-Layer Meteorol 121:33-65

Blackadar AK (1997) Turbulence and diffusion in the atmosphere. Springer, Berlin, $185 \mathrm{pp}$

Chen F, Avissar R (1994) The impact of land-surface wetness heterogeneity on mesoscale heat fluxes. J Appl Meteorol 33:1323-1340

Courault D, Drobinski P, Brunet Y, Lacarrere P, Talbot C (2007) Impact of surface heterogeneity on a buoyancy-driven convective boundary layer in light winds. Boundary-Layer Meteorol 124:383-403

Deardorff JW (1974) Three-dimensional numerical study of the height and mean structure of a heated planetary boundary layer. Boundary-Layer Meteorol 7:81-106

Fesquet C, Dupont S, Drobinski P, Dubos T, Barthlott C (2009) Impact of terrain heterogeneity on coherent structure properties: numerical approach. Boundary-Layer Meteorol 133:71-92

Foken T, Mauder M et al (2009) Energy balance closure for the LITFASS-2003 experiment. Theor Appl Climatol. doi:10.1007/s00704-009-0216-8

Gopalakrishnan SG, Avissar R (2000) An LES study of the impacts of land surface heterogeneity on dispersion in the convective boundary layer. J Atmos Sci 57:352-371

Hadfield MG, Cotton WR, Pielke RA (1991) Large-eddy simulations of thermally forced circulations in the convective boundary layer. Part I: A small-scale circulation with zero wind. Boundary-Layer Meteorol 57:79-114

Hadfield MG, Cotton WR, Pielke RA (1992) Large-eddy simulations of thermally forced circulations in the convective boundary layer. Part II: The effect of change in wavelength and wind speed. Boundary-Layer Meteorol 58:307-327

Hechtel LM, Moeng CH, Stull RB (1990) The effects of nonhomogeneous surface fluxes on the convective boundary layer: a case study using large-eddy simulation. J Atmos Sci 47:1721-1741

Heinemann G, Kerschgens M (2006) Simulation of surface energy fluxes using high-resolution non-hydrostatic simulations and comparisons with measurements for the LITFASS-2003 experiment. BoundaryLayer Meteorol 121:195-220

Huang HY, Margulis SA (2009) On the impact of surface heterogeneity on a realistic convective boundary layer. Water Resour Res 45:w04425. doi:10.1029/2008WR007175

Kang SL (2009) Temporal oscillations in the convective boundary layer forced by mesoscale surface heat-flux variations. Boundary-Layer Meteorol 132:59-81

Kang SL, Davis KJ (2008) The effects of mesoscale surface heterogeneity on the fair-weather convective atmospheric boundary layer. J Atmos Sci 65:3197-3213

Kustas WP, Hatfield JL, Prueger JH (2005) The soil moisture-atmosphere coupling experiment (SMACEX): background, hydrometeorological conditions and preliminary findings. J Hydrometeorol 6:791-804

Letzel MO, Raasch S (2003) Large eddy simulation of thermally induced oscillations in the convective boundary layer. J Atmos Sci 60:2328-2341

Letzel MO, Krane M, Raasch S (2008) High resolution urban large-eddy simulation studies from street canyon to neighbourhood scale. Atmos Environ 42:8770-8784

Lilly DK (2002) Entrainment into mixed layers. Part I: Sharp-edged and smoothed tops. J Appl Meteorol 59:3340-3352

Liu G, Sun J, Yin L (2011) Turbulence characteristics of the shear-free convective boundary layer driven by heterogeneous surface heating. Boundary-Layer Meteorol 140:57-71. doi:10.1007/s10546-011-9591-7

Lynn BH, Rind D, Avissar R (1995) The importance of mesoscale circulations generated by subgrid-scale landscape heterogeneities in general circulation models. J Clim 8:191-205

Mauder M, Göckede CLM, Leps JP, Beyrich F, Foken T (2006) Processing and quality control of flux data during LITFASS-2003. Boundary-Layer Meteorol 121:67-88

Patton EG, Sullivan PP, Moeng CH (2005) The influence of idealized heterogeneity on wet and dry planetary boundary layers coupled to the land surface. J Atmos Sci 62:2078-2097 
Prabha TV, Karipot A, Binford MW (2007) Characteristics of secondary circulations over an inhomogeneous surface simulated with large-eddy simulation. Boundary-Layer Meteorol 123:239-261

Raasch S, Etling D (1998) Modeling deep ocean convection: large eddy simulation in comparison with laboratory experiments. J Phys Oceanogr 28:1786-1802

Raasch S, Harbusch G (2001) An analysis of secondary circulations and their effects caused by small-scale surface inhomogeneities using large-eddy simulation. Boundary-Layer Meteorol 101:31-59

Raasch S, Schröter M (2001) PALM - a large-eddy simulation model performing on massively parallel computers. Meteorol Z 10:363-372

Schröter M, Bange J, Raasch S (2000) Simulated airborne flux measurements in a LES generated convective boundary layer. Boundary-Layer Meteorol 95:437-456

Shen S, Leclerc MY (1995) How large must surface inhomogeneities be before they influence the convective boundary layer structure? A case study. Q J R Meteorol Soc 121:1209-1228

Shuttleworth W, Yang ZL, Arain MA (1997) Aggregation rules for surface parameters in global models. Hydrol Earth Syst Sci 1:217-226

Steinfeld G, Letzel MO, Raasch S, Kanda M, Inagaki A (2007) Spatial representativeness of single tower measurements and the imbalance problem with eddy-covariance fluxes: results of a large-eddy simulation study. Boundary-Layer Meteorol 123:78-98

Steinfeld G, Raasch S, Markkanen T (2008) Footprints in homogeneously and heterogeneously driven boundary layers derived from a Lagrangian stochastic particle model embedded into large-eddy simulation. Boundary-Layer Meteorol 129:225-248

Stull RB (1988) An introduction to boundary layer meteorology. Kluwer, Dordrecht, 666 pp

Sullivan PP, Moeng CH, Stevens B, Lenschow DH, Mayor SD (1998) Structure of the entrainment zone capping the convective atmospheric boundary layer. J Atmos Sci 55:3042-3064

Uhlenbrock J, Raasch S, Hennemuth B, Zittel P, Meijninger WML (2004) Effects of land surface heterogeneities on the boundary layer structure and turbulence during LITFASS-2003: large-eddy simulations in comparison with turbulence measurements. In: 6th Symposium on boundary layers and turbulence. American Meteorological Society, Portland (Maine), paper 9,3

van Heerwaarden CC, de Arellano JVG (2008) Relative humidity as an indicator for cloud formation over heterogeneous land surfaces. J Atmos Sci 65:3263-3277

Weckwerth TM, Parsons DB et al (2004) An overview of the international $\mathrm{H}_{2} \mathrm{O}$ project (IHOP_2002) and some preliminary highlights. Bull Am Meteorol Soc 85:253-277

Wyngaard JC, Coté OR (1974) The evolution of a convective planetary boundary layer-a higher-order-closure model study. Boundary-Layer Meteorol 7:289-308 